\title{
Neuronal Glutamatergic Synaptic Clefts Alkalinize Rather Than Acidify during Neurotransmission
}

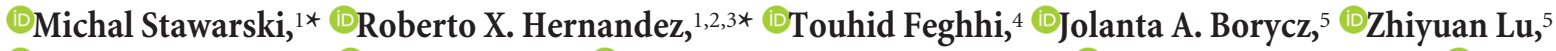 \\ ${ }^{\oplus}$ Andrea B. Agarwal, ${ }^{6}{ }^{-}$Kelly D. Reihl, ${ }^{6}{ }^{\circ}$ Rubens Tavora, ${ }^{1,2}$ A.W.C. Lau, ${ }^{4}$ Ian A. Meinertzhagen, ${ }^{5}$ - Robert Renden, ${ }^{6}$ \\ and $\odot$ Gregory T. Macleod ${ }^{1,2,7,8}$ \\ ${ }^{1}$ Wilkes Honors College, Florida Atlantic University, Jupiter, Florida 33458, ${ }^{2}$ Jupiter Life Science Initiative, Florida Atlantic University, Jupiter, Florida \\ 33458, ${ }^{3}$ Integrative Biology \& Neuroscience Graduate Program, Florida Atlantic University, Jupiter, Florida 33458, ${ }^{4}$ Department of Physics, College of \\ Science, Florida Atlantic University, Boca Raton, Florida 33431, ${ }^{5}$ Department of Psychology \& Neuroscience, Dalhousie University, Halifax, Nova Scotia \\ B3H 4R2, Canada, ${ }^{6}$ Physiology and Cell Biology, University of Nevada, Reno School of Medicine, Reno, Nevada 89557, ${ }^{7}$ Department of Biological Sciences, \\ Florida Atlantic University, Jupiter, Florida 33458, and ${ }^{8}$ Brain Institute, Florida Atlantic University, Jupiter, Florida 33458
}

The dogma that the synaptic cleft acidifies during neurotransmission is based on the corelease of neurotransmitters and protons from synaptic vesicles, and is supported by direct data from sensory ribbon-type synapses. However, it is unclear whether acidification occurs at non-ribbon-type synapses. Here we used genetically encoded fluorescent $\mathrm{pH}$ indicators to examine cleft $\mathrm{pH}$ at conventional neuronal synapses. At the neuromuscular junction of female Drosophila larvae, we observed alkaline spikes of over 1 log unit during fictive locomotion in vivo. Ex vivo, single action potentials evoked alkalinizing $\mathrm{pH}$ transients of only $\sim 0.01$ log unit, but these transients summated rapidly during burst firing. A chemical $\mathrm{pH}$ indicator targeted to the cleft corroborated these findings. Cleft $\mathrm{pH}$ transients were dependent on $\mathrm{Ca}^{2+}$ movement across the postsynaptic membrane, rather than neurotransmitter release per se, a result consistent with cleft alkalinization being driven by the $\mathrm{Ca}^{2+} / \mathrm{H}^{+}$antiporting activity of the plasma membrane $\mathrm{Ca}^{2+}$-ATPase at the postsynaptic membrane. Targeting the $\mathrm{pH}$ indicators to the microenvironment of the presynaptic voltage gated $\mathrm{Ca}^{2+}$ channels revealed that alkalinization also occurred within the cleft proper at the active zone and not just within extrasynaptic regions. Application of the $\mathrm{pH}$ indicators at the mouse calyx of Held, a mammalian central synapse, similarly revealed cleft alkalinization during burst firing in both males and females. These findings, made at two quite different non-ribbon type synapses, suggest that cleft alkalinization during neurotransmission, rather than acidification, is a generalizable phenomenon across conventional neuronal synapses.

Key words: glutamatergic; pH imaging; synaptic cleft; synaptic plasticity

Significance Statement

Neurotransmission is highly sensitive to the $\mathrm{pH}$ of the extracellular milieu. This is readily evident in the neurological symptoms that accompany systemic acid/base imbalances. Imaging data from sensory ribbon-type synapses show that neurotransmission itself can acidify the synaptic cleft, likely due to the corelease of protons and glutamate. It is not clear whether the same phenomenon occurs at conventional neuronal synapses due to the difficulties in collecting such data. If it does occur, it would provide for an additional layer of activity-dependent modulation of neurotransmission. Our findings of alkalinization, rather than acidification, within the cleft of two different neuronal synapses encourages a reassessment of the scope of activity-dependent $\mathrm{pH}$ influences on neurotransmission and short-term synaptic plasticity.

\section{Introduction}

Chemical synapses are built such that some proteins essential for neurotransmission, including voltage gated $\mathrm{Ca}^{2+}$ channels

Received July 25, 2019; revised Jan. 14, 2020; accepted Jan. 15, 2020.

Author contributions: M.S., R.X.H., T.F., A.W.C.L., I.A.M., R.R., and G.T.M. designed research; M.S., R.X.H., J.A.B., Z.L., A.B.A., K.D.R., T.F., R.T., A.W.C.L., R.R., and G.T.M. performed research; M.S., R.X.H., J.A.B., T.F., R.T., A.W.C.L.,

I.A.M., R.R., and G.T.M. analyzed data; M.S., R.X.H., T.F., I.A.M., R.R., and G.T.M. edited the paper; M.S., R.R., and G.T.M. wrote the paper.

This work was supported by National Institutes of Health National Institute of Neurological Disorders and Stroke Award NS103906 to G.T.M. The late Karyl I. Minard helped with construct design and cloning.
(VGCCs) and ligand-gated receptors, are partially exposed to the extracellular milieu of the synaptic cleft. VGCCs, glutamate receptors, and GABA receptors are $\mathrm{pH}$-sensitive at their extracellular aspect (Tang et al., 1990; Barnes and Bui, 1991; Traynelis and
The authors declare no competing financial interests.

*M.S. and R.X.H. contributed equally to this work.

Correspondence should be addressed to Gregory T. Macleod at macleodg@fau.edu.

M. Stawarski's present address: Department of Biomedicine, University of Basel, Basel, 4056, Switzerland.

https://doi.org/10.1523/JNEUROSCI.1774-19.2020

Copyright $\odot 2020$ the authors 
Cull-Candy, 1991; Palmer et al., 2003; Zhou et al., 2007; Chen and Huang, 2014), while acid-sensing ion-channels are also found at synapses and are $\mathrm{pH}$-sensitive by definition (Wemmie et al., 2013; Du et al., 2014). It follows, therefore, that neurotransmission itself is sensitive to changes in extracellular $\mathrm{pH}$ (DeVries, 2001; Palmer et al., 2003; Davenport et al., 2008; Fahrenfort et al., 2009; Sandstrom, 2011; Du et al., 2014; Vroman et al., 2014), and this results in neurological consequences during acid-base disturbances (Chesler, 2003; Obara et al., 2008; Sinning and Hübner, 2013; Wemmie et al., 2013; Zhao et al., 2016). If neurotransmission itself is capable of driving $\mathrm{pH}$ change in the cleft, then implications arise for short-term synaptic plasticity (STSP).

Without knowing the magnitude, polarity, and time course of such $\mathrm{pH}$ changes, there is little prospect of a complete understanding of STSP. The notion that protons $\left(\mathrm{H}^{+}\right)$released from synaptic vesicles (SVs) acidify the cleft receives strong support from imaging data collected from synapses between zebrafish cone photoreceptors and horizontal cells (Wang et al., 2014; Beckwith-Cohen et al., 2019), and electrophysiological studies at sensory ribbon synapses in other vertebrates (DeVries, 2001; Palmer et al., 2003; Hirasawa et al., 2012; Cho and von Gersdorff, 2014; Vincent et al., 2018). However, ribbon synapses exhibit an architecture distinct from conventional neuronal synapses, and release neurotransmitters in response to graded membrane potentials (Chakrabarti and Wichmann, 2019). Individual active zones (AZs) at these synapses dock up to 110 SVs and are capable of "unconventionally" high release rates (von Gersdorff et al., 1996), whereas, at most neuronal synapses each AZ "conventionally" releases no more than a single SV in response to a nerve impulse (Stevens, 2003).

In the mammalian hippocampus and cerebellar cortex, two contrasting techniques have provided information on activitydependent $\mathrm{pH}$ changes in the extracellular space: $\mathrm{pH}$-sensitive electrodes (Kraig et al., 1983; Paalasmaa et al., 1994; Gottfried and Chesler, 1996; Fedirko et al., 2006, 2007; Makani and Chesler, 2007, 2010a,b; Makani et al., 2012) and pH-sensitive fluorescent dyes or proteins (Krishtal et al., 1987; Gottfried and Chesler, 1996; Tong et al., 2006; Du et al., 2014). With few exceptions, these techniques reveal stimulus train-evoked alkaline transients that rise over tens of milliseconds and decay over hundreds of milliseconds. However, neither technique yields a $\mathrm{pH}$ signal directly from the cleft, as $\mathrm{pH}$ electrodes are occluded from intact clefts, and signals from fluorescent probes in the cleft will be drowned out by signals from probes in the larger extrasynaptic volume. Nevertheless, support for the notion that the cleft alkalinizes also comes from electrophysiological data collected from postsynaptic NMDARs in the mouse hippocampus (Makani et al., 2012; Chen and Chesler, 2015). Despite these data, the dogma persists that the cleft acidifies due to acid release from SVs.

To allow minimally invasive measurements of cleft $\mathrm{pH}$ at neuronal synapses, where AZs release no more than one SV per action potential (AP), we targeted fluorescent $\mathrm{pH}$ indicators to the cleft of the Drosophila larval neuromuscular junction (NMJ). In both the broadly defined cleft and directly adjacent to VGCCs, we observed alkalinization in response to APs, rather than acidification. The alkaline transients were reconciled with the activity of the plasma membrane $\mathrm{Ca}^{2+}$ ATPase (PMCA) pumping $\mathrm{Ca}^{2+}$ out of synaptic compartments in electroneutral exchange for protons (Thomas, 2009). The mouse calyx of Held synapse partially relies on the PMCA to clear $\mathrm{Ca}^{2+}$ during burst firing (Kim et al., 2005), and we observed that its cleft also alkalinizes during burst firing, suggesting that most synapses using the PMCA will alkalinize.

\section{Materials and Methods}

\section{Creation of $p H u s i o n-E x$ and $p H e r r y-E x$}

pHusion-Ex combines SE-pHluorin (Sankaranarayanan et al., 2000) with FusionRed (Shemiakina et al., 2012) using the 20 amino acid (AA) linker from ClopHensor (RGSASGGGGGLVPRGSASGA) (Arosio et al., 2010). SE-pHluorin fluorescence decreases with a decrease in $\mathrm{pH}$, whereas FusionRed is $\mathrm{pH}$-insensitive near neutral ( $\mathrm{pKa}=4.6$ ) (Shemiakina et al., 2012). FusionRed was cloned from FusionRed-pBAD (Addgene, plasmid \#54677). To create cleft-targeted versions of pHusion, we purchased a biosynthesized DNA backbone cloned into a pJFRC14 plasmid from GenScript. This plasmid enables expression of heterologous genes in fruit fly under the control of GAL4-UAS system (Brand and Perrimon, 1993). The backbone encoded (from $5^{\prime}$ end) a secretion signal sequence from $\operatorname{IgG} \kappa$, the complete SE-pHluorin cDNA, restriction sites enabling the subcloning of FusionRed cDNA, and the transmembrane domain of the human platelet-derived growth factor receptor (hPDGFR). The transmembrane domain was separated from FusionRed using the same 20 AA linker above. FusionRed was cloned in-frame into the backbone. pHerry-Ex, like pHusion-Ex, combines the $\mathrm{pH}$-sensitive SE-pHluorin with an RFP that is $\mathrm{pH}$-insensitive near neutral (mCherry; pKa < 4.5) (Shaner et al., 2004), and it was assembled in the same manner as pHusion-Ex. mCherry was cloned from pcDNA3.3_mCherry (Addgene, plasmid \#26823). pHusion-Ex and pHerry-Ex constructs were sequenced, purified with the QIAGEN Plasmid Maxi Kit, and each injected into $\mathrm{P}\{$ CaryP $\}$ attP2 Drosophila embryos by Rainbow Transgenic Flies. The third chromosome carrying the transgene was balanced opposite the tubby marker/balancer TM6B, $\mathrm{Tb}^{1}$. Single copies of either motor neuron (MN; nSyb-GAL4) or muscle (B24-GAL4) drivers drove expression of pHusion-Ex or pHerry-Ex.

\section{Creation of PMCA-kisser-FAP and Cac-kisser-pHerry-TM}

A plasmid encoding the $\alpha$ FAP gene was purchased from Spectra Genetics, subcloned into a biosynthesized DNA backbone manufactured by GenScript, and then subcloned in the pJFRC14 plasmid. The backbone encoded (from $5^{\prime}$ end) a signal peptide from Wingless, an MCS for $\alpha$ FAP, two protein tags directly upstream and downstream of the MCS (HA-tag and myc-tag, respectively), a transmembrane domain of hPDGFR, and a modified carboxy terminus of Drosophila melanogaster PMCA. The calmodulin $(\mathrm{CaM})$ binding site in the PMCA carboxy terminus was identified in silico, and scrambled to prevent unwanted oligomerization or cleavage by calpains (Di Leva et al., 2008; Benetti et al., 2011). Cac-kisserpHerry-TM was similarly created, except that the calmodulin and PDZ binding domains of the Cac carboxy terminus were scrambled to prevent spurious interactions and proteolytic cleavage. Injection, balancing, and expression were performed as described above. FAP probes were conjugated with a $\mathrm{pH}$-sensitive fluorogen as follows: PM-impermeable seRed-pH dye (Spectra Genetics) was added to the bath of a fillet-dissected female larvae at a final concentration of $100 \mathrm{~nm}$ for $15 \mathrm{~min}$. The full sequences of PMCA-kisser-FAP and Cac-kisser-pHerry-TM is given in the supplementary materials of Stawarski et al. (2018).

\section{Creation of membrane-targeted pseudoratiometric GECIs}

One of the pseudoratiometric genetically-encoded $\mathrm{Ca}^{2+}$ indicators (GECIs) used in this study combines GCaMP6m (Chen et al., 2013) with FusionRed. GCaMP6m was cloned from pGP-CMV-GCaMP6m (Addgene, plasmid \#40754), and a backbone was biosynthesized (Genscript) to facilitate cloning. The backbone encoded (from $5^{\prime}$ end) a myristoylation signal sequence, HA-tag, MCS for the $\mathrm{Ca}^{2+}$-insensitive protein FusionRed, the 20 AA linker described above, and an MCS for the $\mathrm{Ca}^{2+}$ sensitive protein GCaMP6m. Carboxy-terminal localization was chosen for GCaMP6m to limit spatial and rotational constraints. The backbone was cloned into the pJFRC14 plasmid. Injection, balancing, and expression were performed as described above.

A second pseudoratiometric GECI developed for this study, called Coincidence Detector 1.0, combines C-terminal jRGECO1a (Addgene, plasmid \#61563) (Dana et al., 2016) with iRFP670 (Addgene, plasmid \#45457) (Shcherbakova and Verkhusha, 2013) in the cytosol, and this was combined through the hPDGFR transmembrane domain with SEpHluorin to enable simultaneous $\mathrm{pH}$ reporting in the synaptic cleft. The 
backbone was cloned into the pJFRC14 plasmid. Injection, balancing, and expression were performed as described above.

\section{Preparation of fillet-dissected larval preparations for imaging}

Female third instar Drosophila larvae were fillet-dissected in chilled Schneider's insect medium (Sigma Millipore, catalog \#S0146). All except the segmental nerves of interest were cut from the ventral ganglion. The ventral ganglion was then cut just posterior to the brain hemispheres, and the brain hemispheres and head of the larva were removed. A loop of a segmental nerve projecting into the fourth abdominal hemisegment was then drawn into the lumen of a glass micropipette (Sigma Millipore, catalog \#HT10132) while the nerve was still connected to the remaining fragment of the ventral ganglion containing somata of the MNs that project to segment 4 . The Schneider's medium was then replaced with HL3 (Stewart et al., 1994) or HL6 physiological solution (Macleod et al., 2002). The preparation was allowed to equilibrate for $10 \mathrm{~min}$ before imaging under the microscope.

In vivo imaging was performed as described previously (Rossano et al., 2013). The average $\mathrm{pH}$ value in the initial $5 \mathrm{~s}$ of the recordings was treated as baseline and used to calculate $\Delta \mathrm{pH}$ values.

\section{Wide-field imaging and electrical stimulation of GEpHI- and} GECI-expressing larvae

Wide-field microscopy was performed on a Nikon Eclipse FN1 microscope fitted with a $100 \times$ water-immersion (1.1 NA) objective. Preparations were illuminated with a Lumencor SPECTRA-X light source, and emitted light was captured with two Andor iXon3 CCD cameras (DU897) mounted on an Andor TuCam beam-splitter. Separate emission wavelengths were collected by separate cameras, with sequential excitation of fluorophores used to minimize channel cross talk. Imaging and electrical stimulation were controlled through PC running Nikon NIS Elements-AR software. Timing of electrical stimulation was controlled by a Master- 8 controller (A.M.P.I.) triggered by PC software. The pulses were delivered to the nerve itself by a constant voltage stimulator (model DS2A-Mk. II, Digitimer). Each impulse was $300 \mu$ s in duration and 2-3 $\mathrm{V}$ in amplitude. Three categories of experimental protocols were used. (1) AP train experiments: A train of 20 or 40 impulses was delivered to the nerve at 21 or $42 \mathrm{~Hz}$, respectively, $20 \mathrm{~s}$ after the initiation of imaging. (2) Single AP experiments: Experiments were programmed in terms of loops of imaging frames (100 loops of 94 frames). A single AP was delivered before frame 20 of each loop. (3) Paired pulse experiments: These differed from single AP experiments by the addition of an AP that followed $\sim 100 \mathrm{~ms}$ (8 imaging frames) after the first AP.

Fluorescent proteins were excited with $483 / 32 \mathrm{~nm}$ and 550/15 nm light (CWL/FWHM). Emitted light was directed to the two EMCCD cameras via a $580 \mathrm{~nm}$ dichroic mirror through 525/30 nm and 661/20 nm filters. Each ratio of fluorescence intensity was based on two frames recorded in succession. The signal of SE-pHluorin or GCaMP6m was recorded first, with the signal of FusionRed or mCherry recorded second. For analysis and presentation, the ratio was represented as if the two signals were collected at the same time, except where temporal discrimination was critical (see Fig. 4I).

Imaging of stimulus train-evoked $\mathrm{pH}$ transients was performed in bicarbonate-buffered HL3 physiological solution ( $5 \mathrm{~mm} \mathrm{KCl,} 20 \mathrm{~mm}$ $\mathrm{MgCl}_{2}, 70 \mathrm{~mm} \mathrm{NaCl}, 115 \mathrm{~mm}$ sucrose, $5 \mathrm{~mm}$ trehalose, $15 \mathrm{~mm} \mathrm{NaHCO}_{3}$ ). The solution was then bubbled with $95 \% \mathrm{O}_{2} / 5 \% \mathrm{CO}_{2}$, and $\mathrm{pH}$ adjusted to 7.2. HL3 was prepared in advance, filtered, and frozen to $-80^{\circ} \mathrm{C}$ without delay. On the day of the experiment, an aliquot of the solution was thawed, supplemented with $\mathrm{CaCl}_{2}$ (2 mM final concentration), and used as is (in unbubbled experiments) or constantly bubbled with $95 \%$ $\mathrm{O}_{2} / 5 \% \mathrm{CO}_{2}$ (in bubbled experiments, with or without acetazolamide or HEPES). Muscle contractions were substantially reduced by preincubating the fillet-dissected larvae for $30 \mathrm{~min}$ in bicarbonate-buffered HL3 supplemented with $100 \mu \mathrm{M}$ ryanodine (Enzo Life Sciences) solubilized in DMSO. Carbonic anhydrase (CA) activity was blocked by 30 min preincubation with $10 \mu \mathrm{m}$ acetazolamide (Sigma Millipore) solubilized in DMSO. $\mathrm{pH}$ and $\mathrm{Ca}^{2+}$ transients were elicited by stimulating the preparations with 20 impulses at $21 \mathrm{~Hz}$ and recording the fluorescence from Type Ib boutons located on muscle 6 .
In single AP experiments, the exposure time for each channel was set to $4 \mathrm{~ms}$, and $4 \times 4$ binning was performed to achieve an imaging speed of 94 frames per second (fps) per camera, and therefore 94 ratios per second. Cytosolic $\mathrm{Ca}^{2+}$ levels were imaged using GCaMP6m fused to myristoylated FusionRed. Imaging of single AP-evoked $\mathrm{pH}$ transients in animals expressing PMCA-kisser-FAP was similar to other single AP experiments, with the following differences: after incubation with the fluorogen (se-RedpH) NMJs were sequentially excited with 549/15 nm and 640/30 nm light, and emission at 705/70 nm was recorded with a single Andor iXON3 CCD camera. Pixels were $4 \times 4$ binned to increase the signal-to-noise ratio and achieve an imaging speed of 94 ratios $(549 / 640 \mathrm{~nm})$ per second.

Live in situ imaging was performed on Type Ib boutons on muscle 6 of segment 4 in HL3 solution. The location of MN terminals could be visualized at live NMJs, subsequent to GECI or genetically-encoded $\mathrm{pH}$ indicator (GEpHI) imaging, using the DyLight405-conjugated AffiniPure goat antibody for HRP (Jackson ImmunoResearch Laboratories, catalog \#123-475-021) after $30 \mathrm{~min}$ incubation using a dilution of 1:200 at room temperature.

\section{Imaging data analysis}

Image analysis was performed in ImageJ. Movement of the NMJ during imaging was accounted for by aligning consecutive frames to the first one using the Template Matching plugin written by Qingzong Tseng. ROIs were drawn to cover an entire bouton(s); a reference ROI (background) and the mean pixel intensity values were calculated within each channel for each frame; and the numerical data were exported to Microsoft Excel. Each frame was background adjusted by subtracting the mean intensity value of the reference ROI from the mean intensity value of the bouton(s) ROI. For single AP experiments, the experiment was looped 100 times $(n=100)$ for each $\mathrm{NMJ}(N=1)$, and the loops were averaged.

Decay time constants in single AP experiments were calculated by fitting a monoexponential curve $r=y_{0}+a \mathrm{e}^{-\mathrm{t} / \tau}$ to the decay curve of the $\mathrm{pH}$ transient. The amplitude was calculated by subtracting the peak displacement of a moving average from the prestimulus baseline value: 3 and 17 point moving averages when sampling at 94 and $560 \mathrm{~Hz}$, respectively. The baseline value was calculated as the average value in the $100 \mathrm{~ms}$ period before the stimulus. The amplitude in response to trains was calculated by subtracting the peak of a moving average from the value of the baseline in the $500 \mathrm{~ms}$ before the stimulus: 5 or 9 point moving averages when sampling at 10 and $19 \mathrm{~Hz}$, respectively.

Curve fitting was performed in SigmaPlot 10. If there was no acceptable fit of an exponential curve (large values of $\tau$ ), a linear approximation was used instead, in accordance with Taylor's theorem as follows:

$$
e^{-x / \tau}=\sum_{n=0}^{\infty}(-x / \tau)^{n} / n !, \text { so for } \mathrm{b} \rightarrow 0 e^{-b x} \approx 1-x / \tau
$$

\section{Experimental design and statistical analysis}

Female Drosophila larvae and mice of both sexes were used in this study. Statistical analyses were performed in SigmaStat 3.5. Pairwise comparisons were performed using Student's $t$ tests, whereas comparisons between multiple groups were performed by one-way ANOVA; details are given in each instance. Data describing single AP-evoked $\mathrm{pH}$ transients are expressed as mean \pm SD as the product of each experiment $(N=1)$ is a trace, the arithmetic mean of 100 traces $(n=100)$, which is then analyzed to give single estimates of various parameters. Data describing train-evoked $\mathrm{pH}$ transients at the calyx of Held are expressed as mean \pm SEM. An estimate of each parameter $(N=1)$ is based on 3 trains $(n=3)$ with a nonstimulus control between each to provide a measure of bleaching.

\section{Immunohistochemistry of Cac-kisser-pHerry-TM}

Larvae expressing the Cac-kisser-pHerry-TM construct were fillet dissected in chilled Schneider's media and washed with HL3 $\left(20 \mathrm{~mm} \mathrm{Mg}^{2+}\right.$, no added $\left.\mathrm{Ca}^{2+}\right)$. Preparations were then fixed in Bouin's solution (Sigma Millipore, catalog \#HT10132) for $1 \mathrm{~min}$ at room temperature, washed $(4 \times 10 \mathrm{~min})$ with PBS, $\mathrm{pH} 7.2$, then permeabilized with $1 \%$ PBST (PBS containing 1\% Triton X-100) for $1 \mathrm{~h}(4 \times 15 \mathrm{~min})$ and blocked for $30 \mathrm{~min}$ in PBST containing $0.2 \%$ Triton X-100, 2\% BSA, and $5 \%$ goat serum (Sigma Millipore, catalog \#G9023). Preparations were immunolabeled by incubating with primary antibodies overnight at $4^{\circ} \mathrm{C}$ : 
rabbit AF488-conjugated anti-GFP (1:500, Invitrogen, catalog \#A21311) and mouse anti-Bruchpilot (1:300, nc82; DSHB Hybridoma Bank). The following day, preparations were rinsed with $0.2 \%$ Triton PBST for $1 \mathrm{~h}$ $(4 \times 15 \mathrm{~min})$, preparations were then incubated at room temperature for $1 \mathrm{~h}$ with anti-mouse AF555 (Invitrogen, catalog \#A21127) and Dylight405conjugated anti-HRP (1:500, Jackson ImmunoResearch Laboratories, catalog \#123475021). Preparations were finally washed with $0.2 \%$ Triton PBST for $1 \mathrm{~h}(4 \times 15 \mathrm{~min})$, mounted on glass slides with antifade reagent (SlowFade Gold, catalog \#S36937, Invitrogen), and covered with 0.16$0.19 \mathrm{~mm}$ coverslips (Corning, catalog \#2870-22). Series of images were collected from muscle fiber \#6, MN6/7-Ib with a $100 \times 1.3$ NA oil objective on a Nikon A1 confocal microscope fitted with GaAsP detectors. Boutons were scanned sequentially starting with the longest wavelength and progressing to the shortest (561, 488, and $405 \mathrm{~nm}$ ). Bouton images represent collapsed $Z$ series that encompass the entire depth of the bouton ( $\sim 10$ sections, $0.25 \mu \mathrm{m}$ between slices). No bleed-through to other channels could be detected when tested with single fluorophores. A pinhole size 1 airy unit for AF555 imaging was used for the three imaging channels.

\section{In situ calibration of $p H$ indicators}

pHerry, a fusion construct between SE-pHluorin and mCherry using the same linker as pHusion, was previously calibrated (Rossano et al., 2017). Therefore, instead of a full calibration of pHusion-Ex, only the linear response range of pHluorin-Ex was calibrated, $\sim \pm 0.5 \mathrm{pH}$ units on either side of the $\mathrm{pK}_{\mathrm{a}}$ of SE-pHluorin $\sim 7.11$ (Sankaranarayanan et al., 2000). Third instar larvae were fillet dissected, incubated for 30 min in HL6 solution with the appropriate $\mathrm{pH}$, and imaged under a wide-field microscope. The fluorescence intensity ratio of postsynaptically expressed pHusion-Ex was calculated from $1004 \mathrm{~ms}$ exposure pairs. Following imaging, the larvae were washed thrice with HL6, and the procedure was repeated until imaging was performed in every preselected $\mathrm{pH}$. The order of presentation of $\mathrm{pH}$-adjusted HL6 was pseudorandomized (see Fig. 1E).

The $\mathrm{pH}$-sensitive fluorogen bound to PMCA-kisser-FAP was similarly calibrated. Larvae were preincubated with se-Red-pH diluted in HL6 for 30 min to label the PMCA-kisser-FAP. Calibration then proceeded using a protocol similar to that used to calibrate $\mathrm{pHusion}-\mathrm{Ex}$ with $\mathrm{pH}$-adjusted HL6 solutions spanning a range from 6.56 to 7.82 (see Fig. $3 F$ ).

GEpHI calibration is not possible under in vivo conditions, due to an inability to control $\mathrm{pH}$ in vivo, and so in vivo $\mathrm{pH}$ changes in the synaptic cleft were calibrated with data collected during in situ experiments. Due to the large changes in ratio in vivo, the partial calibration in situ (linear) will only provide an approximate calibration for $\mathrm{pH}$ relative to the pHusion-Ex ratio.

\section{Drosophila electron microscopy}

Neuromuscular junctions of female third instar larvae were prepared for electron microscopy as previously reported (Lu et al., 2016).

\section{Creation of cleft and cytosolic GEpHIs for the mouse}

pHusion-Ex and cyto-pHusion were subcloned into a plasmid (pAM) containing adeno-associated virus 2 inverted terminal repeats flanking a cassette consisting of the $1.1 \mathrm{~kb}$ cytomegalovirus enhancer/chicken $\beta$-actin promoter, the woodchuck post-transcriptional regulatory element, and the bovine growth hormone polyA, described previously (Groh et al., 2008). Serotype rAAV9 viruses were custom packaged by Vigene Biosciences. Viral titers were $>1 \times 10^{13} \mathrm{GC} / \mathrm{ml}$.

\section{Mouse brain slice preparation and imaging at the calyx of Held}

Ethical approval. All animals in this study were used in accordance with animal welfare protocols approved by the University of Nevada, Reno and according to U.S. standards for animal research (National Research Council, 2011), to minimize the animal's pain and distress. WT C57 Bl/6 mice were obtained from Charles River Laboratories (C57BL/6NCrl, strain code 027), or used from a colony maintained locally. Animals were given ad libitum access to water and were maintained on standard mouse chow. Animals of both sexes were used at P21-P28 for the experiments listed below. In total, 8 animals were used in this study.

Targeted viral infection. CNS injection of neonatal mice followed protocols described previously (Singh et al., 2018). Briefly, 1-d-old (P1) mice were chilled on ice for $>5$ min until unresponsive, then fixed on a stereotaxic surgery platform. The mice were injected with $2 \mu \mathrm{l}$ of rAAVpHusion-Ex or rAAV-cyto-pHusion in sterile PBS, using empirically determined stereotaxic coordinates targeting the ventral cochlear nucleus $(\mathrm{VCN})$. Subsequently, mice were put on a heating pad $\left(37^{\circ} \mathrm{C}\right)$ to recover, then returned to their home cage.

Acute brain slice preparation. Animals were rapidly decapitated, and $200-\mu \mathrm{m}$-thick transverse brainstem slices containing the medial nucleus of the trapezoid body (MNTB) and VCN were prepared using a vibratome (VT 1200S, Leica Microsystems), as previously described (Lujan et al., 2016). Slicing solution contained the following (in mM): $85 \mathrm{NaCl}$, $2.5 \mathrm{KCl}, 25$ glucose, $25 \mathrm{NaHCO}_{3}, 1.25 \mathrm{NaH}_{2} \mathrm{PO}_{4}, 75$ sucrose, $0.5 \mathrm{CaCl}_{2}, 7$ $\mathrm{MgCl}_{2}, 3$ myo-inositol, 2 sodium pyruvate, 0.4 ascorbic acid, $\mathrm{pH} 7.4$, when continuously bubbled with carbogen gas $\left(95 \% \mathrm{O}_{2} / 5 \% \mathrm{CO}_{2}\right)$. Slices were transferred to an incubation chamber containing low-bicarbonate ACSF (in mm as follows): $125 \mathrm{NaCl}, 2.5 \mathrm{KCl}, 25$ glucose, $10 \mathrm{NaHCO}_{3}$, $1.25 \mathrm{NaH}_{2} \mathrm{PO}_{4}, 2 \mathrm{CaCl}_{2}, 1 \mathrm{MgCl}_{2}, 3$ myo-inositol, 2 sodium pyruvate, 0.4 ascorbic acid, 315-320 mOsm density, bubbled with carbogen gas for 45 min, then used within $4 \mathrm{~h}$.

Imaging. Recordings were performed in low-bicarbonate ACSF, plus 5 mM HEPES where indicated, at $35^{\circ} \mathrm{C}$. Recording chamber had $\sim 800 \mu \mathrm{l}$ volume and was continuously perfused. An AXIO Examiner A1 upright microscope (Carl Zeiss) with a $60 \times$ water-immersion objective (1.0 NA) was used for visualization. Infected terminals were identified by Fusion Red fluorescence in the calyx terminal, and SE-pHluorin fluorescence confirmed. Illumination and camera triggering were controlled by an Axon $1440 \mathrm{AD}$ board using pClamp 10.0 software. Images were captured on a Hamamatsu EM-CCD camera (ImagEM X2) controlled by HCI Image software (RRID:SCR_015041).

Images of GEpHI fluorescence responses to changes in superfusate $\mathrm{pH}$ were collected at room temperature $\left(23^{\circ} \mathrm{C}-25^{\circ} \mathrm{C}\right)$ with bath perfusion rates of $\sim 10-12$ volumes per minute. Wide-field fluorescence illumination was provided by a Polychrome II monochromator (TILL Photonics). Illumination alternated between $470 / 50 \mathrm{~nm}$ excitation for SEpHluorin and 550/50 $\mathrm{nm}$ excitation for FusionRed, and was cleaned up with 488/12.5 and 555/13 nm filters, respectively. Emission light was captured through 521/13 and 598/22 nm filters, respectively (Quad-band filter set 89401, Chroma Technology). Images were captured with $241 \mathrm{~ms}$ exposure at an EM Gain setting of 200, with one frame captured every 250 $\mathrm{ms}$ to provide image pairs at $2 \mathrm{~Hz}$. One fluorescence ratio was generated for each image pair.

For changes in $\mathrm{pH}$ due to synaptic activity, bath perfusion was $1-2$ $\mathrm{ml} / \mathrm{min}$, and heated to $33^{\circ} \mathrm{C}-35^{\circ} \mathrm{C}$ with an inline heater (Warner Instruments). Midline stimulation $(400 \mathrm{~Hz}, 1 \mathrm{~s})$ was delivered via a bipolar electrode using a Grass S88 Stimulator. Excitability of each infected terminal was validated before optical recordings by juxtacellular spike recordings (data not shown), and stimuli were delivered at $0.5 \mathrm{~V}$ above threshold. Illumination was provided by an LED light engine (Lumencor, SOLA), mitigated to $20 \%$ intensity with a neutral density filter. SEpHluorin was excited using a $470 / 40 \mathrm{~nm}$ filter and a $495 \mathrm{~nm}$ beam splitter, and emission was collected through a 525/50 nm filter. Images were acquired at $25 \mathrm{~Hz}$ using $2 \times$ pixel binning, and $39 \mathrm{~ms}$ exposures. Electron microscopy sensitivity gain was set to $50 \times$. Usually, stimulation trains were looped three times per cell with $>1$ min between loops in ACSF lacking HEPES. In many cases, the same cell was then perfused with HEPES buffer ( $>10 \mathrm{~min})$, and then imaged as before.

Images of calyx GEpHI fluorescence were analyzed with HCI Image (RRID:SCR_015041). Briefly, an ROI was drawn to contain the presynaptic calyx terminal, and the mean fluorescence for each image was reported as change from baseline $\left(\Delta \mathrm{F} / \mathrm{F}_{\text {base }}, \%\right)$, where baseline was determined as the average signal 2-3 s before stimulation. Responses were corrected for bleaching and other inhomogeneities in camera function by subtracting interleaved illumination controls lacking electrical stimulus.

Transmission electron microscopy. Mice 18-d-old were transcardially perfused with PBS $+100 \mathrm{U} / \mathrm{ml}$ heparin, then Karnovsky's fixative (2\% PFA, $1.5 \%$ glutaraldehyde, $0.1 \mathrm{~m}$ sodium cacodylate buffer). The brain was removed and postfixed for $24-48 \mathrm{~h}$ in Karnovsky's fixative. The MNTB was dissected from tissue sliced at $200 \mu \mathrm{m}$ in $0.1 \mathrm{M}$ cacodylate buffer and postfixed with $2 \% \mathrm{OsO}_{4}$ in cacodylate buffer. The tissue was 


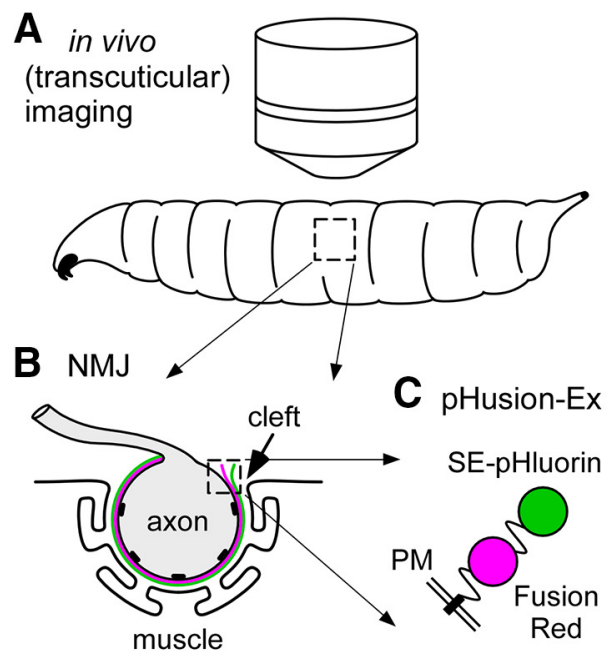

D

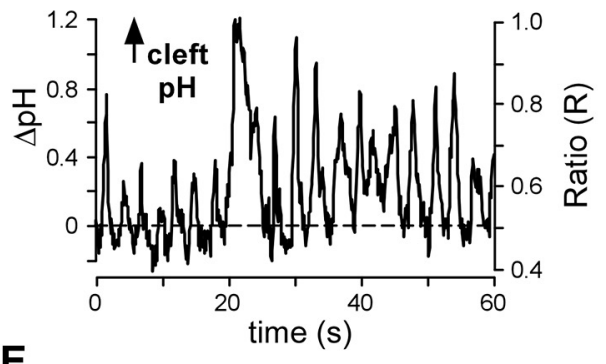

$\mathbf{E}$

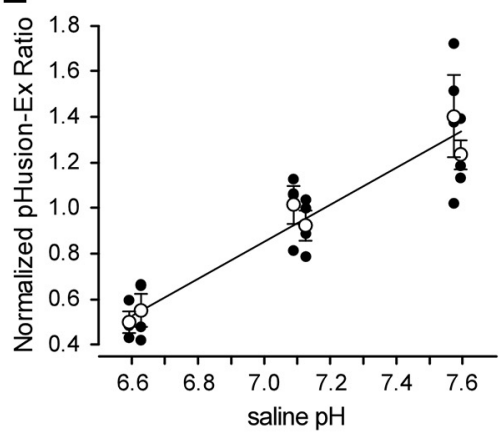

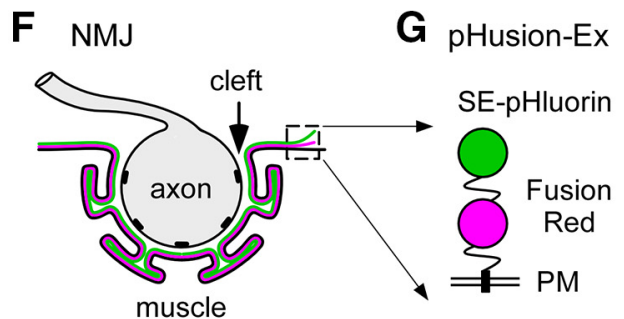

H
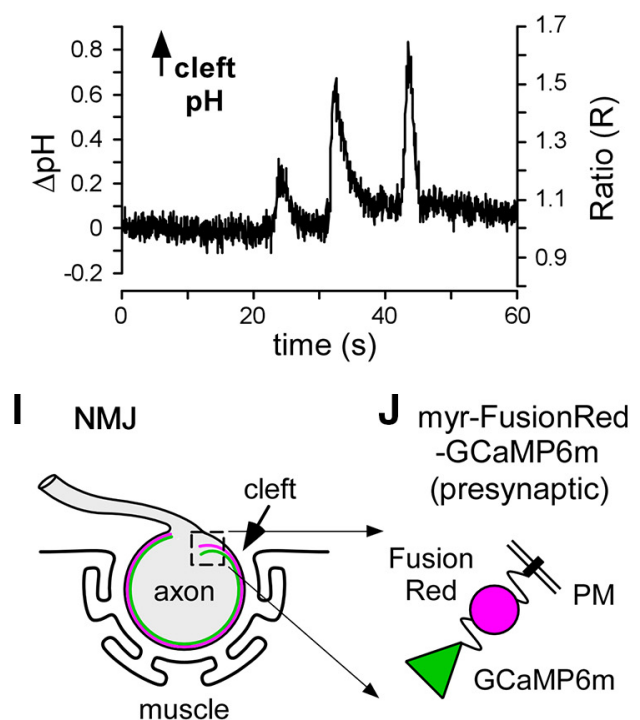

K

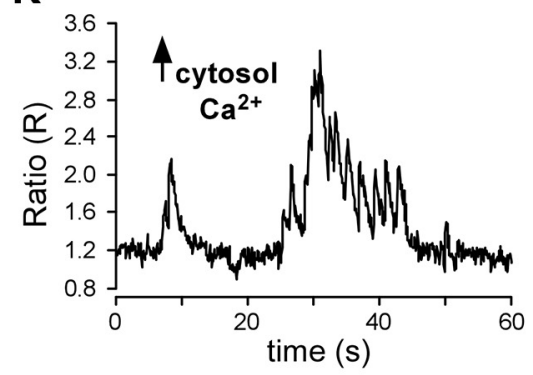

Figure 1. Trans-cuticular imaging of pHusion-Ex at the in vivo NMJ reveals synaptic cleft alkalinization during endogenous activity. $A$, A restrained third instar larva (between a glass slide and a coverslip; data not shown) under a $20 \times$ objective. $\boldsymbol{B}$, NMJ cross section showing the cleft distribution of pHusion-Ex expressed using the MN driver nSyb-GAL4. C, pHusion-Ex fluorophores localized to the cleft through sequential $\mathrm{C}$ terminus to $\mathrm{N}$ terminus fusions of SE-pHluorin to FusionRed to the hPDGFR transmembrane domain. $\boldsymbol{D}$, The ratio of changes in the fluorescence intensity of pH-sensitive SE-pHluorin relative to $\mathrm{pH}$-insensitive FusionRed at an individual MN terminal (right-hand ordinate), revealing cleft alkalinization and reflecting spontaneous motor activity of fictive locomotion. Ratio changes are represented as $\mathrm{pH}$ changes on the left-hand ordinate. $E$, Calibration curve (in situ) for pHusion-Ex. Plotted values normalized to pH 7.2. F, NMJ cross section showing the cleft distribution of pHusion-Ex expressed using the muscle driver 24B-GAL4. Boutons of the axon terminal are embedded in the muscle which forms postsynaptic folds around the bouton. $G$, pHusion-Ex fluorophores localized to the cleft as in C. H, Trans-cuticular imaging of postsynaptically expressed pHusion-Ex at an individual NMJ reveals spontaneous cleft alkalinization of the synaptic cleft in vivo. $\boldsymbol{I}-\boldsymbol{K}$, The intracellular $\mathrm{Ca}^{2+}$ reporter myr-FusionRed-GCaMP6m reveals the spontaneous motor activity of fictive locomotion in vivo. $\boldsymbol{I}$, NMJ cross section, showing the distribution of GCaMP6m and FusionRed expressed in MNs (nSyb-GAL4). J, The C terminus of GCaMP6m was fused to the N terminus of myristoylated FusionRed. $\boldsymbol{K}$, Spontaneous changes in the fluorescence intensity of GCaMP6m relative to FusionRed. An increase in the fluorescence ratio (GCaMP6m/FusionRed) represents an increase in presynaptic $\mathrm{Ca}^{2+}$ levels. $\boldsymbol{D}, \boldsymbol{H}, \boldsymbol{K}$, Fluorophore ratio measurements collected at $4.3 \mathrm{~Hz}$.

dehydrated in an ethanol series $(50 \%, 70 \%, 100 \%)$, further dehydrated with propylene oxide, and then embedded in Spurr's resin. Ultrathin sections containing the MNTB were cut on an ultramicrotome (Leica Microsystems, Ultracut) and stained with 3\% uranyl acetate and 3\% lead citrate. Images were acquired at 7900-46,000 magnification on a Philips CM10 transmission electron microscope.

\section{Results}

The synaptic cleft shows robust alkalinization in vivo during spontaneous activity

We have previously observed spontaneous acidification of MN terminals in vivo through the cuticle of intact but restrained Dro- sophila larvae (Rossano et al., 2013). Changes in cytosolic pH, presumably reflecting motor patterns, were transduced into a change in fluorescence emitted from GEpHIs within the MN cytosol. To determine whether the synaptic cleft of the NMJ undergoes $\mathrm{pH}$ changes under the same conditions, we used a ratiometric GEpHI called "pHusion-Ex" (superecliptic-pHluorin fused to FusionRed) localized to the extracellular surface of plasma membranes (Fig. 1A-C). pHusion-Ex was expressed in MNs, and both fluorophores were visible at NMJs through the cuticle. $\mathrm{pH}$-sensitive SE-pHluorin fluorescence was highly dynamic while $\mathrm{pH}$-insensitive FusionRed showed little change, and 
the fluorescence ratio is largely insulated from movements that accompany motor patterns. Sharp elevations off the baseline indicate alkalinization of the cleft (Fig. 1D). Subsequent ex vivo calibrations (Fig. $1 E$ ) indicated that the cleft cycles through a substantial $\mathrm{pH}$ range in vivo, at times exceeding 1 log unit (Fig. 1D). Similar alkalinizing transients were observed at the NMJ when pHusion-Ex was expressed in the muscle (Fig. $1 F-H$ ). These alkaline transients likely reflect bursts of motor activity as trans-cuticular imaging of a ratiometric $\mathrm{Ca}^{2+}$ indicator in $\mathrm{MN}$ terminals shows similarly volatile transients (Fig. $1 I-K$ ).

\section{Single APs evoke rapid alkalinizing transients in the cleft}

To investigate the basis of synaptic cleft $\mathrm{pH}$ change during individual APs, we examined the ex vivo larval NMJ preparation during nerve stimulation. In response to a single AP, presynaptically expressed pHusion-Ex revealed a rapid alkaline transient within the cleft (Fig. $2 A-C$ ). In physiological $\mathrm{Ca}^{2+}$ levels (2 mM), and with ryanodine present at $100 \mu \mathrm{M}$ to reduce muscle contraction (Sullivan et al., 2000), alkalinization peaks at $0.54 \pm 0.13 \%$ $(\% \Delta \mathrm{F} / \mathrm{F}, \mathrm{SD}, N=5)$ above the baseline ratio $(\Delta \mathrm{pH}: 0.006), 59 \pm$ $11 \mathrm{~ms}(\mathrm{SD})$ after the stimulus, before a rapid return to baseline $(\tau=62 \pm 19 \mathrm{~ms}, \mathrm{SD})$. The Drosophila larval NMJ is characterized by elaborate postsynaptic folds referred to as the subsynaptic reticulum (SSR) (Johansen et al., 1989) (Fig. 2D), and when pHusion-Ex is targeted to the muscle pHusion-Ex appears to occupy the full extent of these folds (Fig. 2E,F). pHusion-Ex also revealed alkalinization within the cleft adjacent to the postsynaptic membrane $(1.00 \pm 0.86 \% \mathrm{SD} ; \Delta \mathrm{pH}: 0.013 ; N=5)$, and a rapid rate of recovery ( $\tau=94 \pm 52 \mathrm{~ms}, \mathrm{SD}$ ) (Fig. $2 G$ ).

Postsynaptic pHusion-Ex in the cleft also allowed for measurements in the absence of ryanodine as measurements of the strong SSR signal were less susceptible to contraction. Alkaline transients appeared to be larger and slower to recover (1.94 \pm 1.31\%, SD; $\Delta \mathrm{pH}$ : 0.025; $\tau=139 \pm 62 \mathrm{~ms}, \mathrm{SD} ; N=13$; Fig. $2 G$ ) than those measured in ryanodine, suggesting an involvement of $\mathrm{Ca}^{2+}$ release from ryanodine receptor channels. However, the data did not support a conclusion of significant differences (amplitude: $p=0.16$; tau: $p=0.18, \mathrm{df}=16$ for each, Student's $t$ tests). All signs of alkalinization were occluded when $\mathrm{Ca}^{2+}$ was omitted from the saline (data not shown). Glutamate, which desensitizes $\mathrm{Ca}^{2+}$-permeable postsynaptic glutamate receptors at the Drosophila NMJ (Heckmann and Dudel, 1998), also occluded alkalinization $(0.03 \pm 0.06 \%, \mathrm{SD} ; N=10$; one-way ANOVA on ranks, Kruskal-Wallis H test, $\mathrm{H}=21.8$, $\mathrm{df}=2, p<0.001$; Dunn's method post hoc test; Fig. $2 H$ ), suggesting that the alkaline transients are linked to $\mathrm{Ca}^{2+}$ movement across the postsynaptic membrane, consistent with the $\mathrm{Ca}^{2+} / \mathrm{H}^{+}$antiporting action of PMCA in the postsynaptic membrane. Aspartate has an almost identical complement of ionizable groups to glutamate but did not occlude alkalinization $(1.15 \pm 0.71 \%$, SD; $\Delta \mathrm{pH}$ : $0.015 ; N=$ 12; Fig. $2 \mathrm{H}$ ), indicating that glutamate does not occlude the $\mathrm{pH}$ transient by acting as a $\mathrm{pH}$ buffer. Glutamate has little effect on the presynaptic $\mathrm{Ca}^{2+}$ transient (Macleod et al., 2004), and so glutamate's complete occlusion of the alkaline transient strongly suggests that $\mathrm{Ca}^{2+}$ pumping from the presynaptic side of the synapse plays a negligible role in cleft alkalinization at the larval NMJ.

A test of the effect of two APs in rapid succession (100 ms interval) revealed that, although single AP-evoked $\mathrm{pH}$ changes are small, they are likely to summate during burst firing (Fig. $2 I-K)$. Notably, in no case was a rapid acidifying transient observed immediately following the stimulus that might have indicated the release of acid from SVs.
The slow sampling speed of the EMCCD camera (94 frames per second), relative to exocytosis, left open the possibility that we missed an acid transient immediately following each AP. Using the high temporal resolution offered by a confocal laserscanning microscope, we scanned across the SSR at 560 lines per second. pHusion-Ex revealed alkaline transients but no signs of acidification immediately following the nerve stimulus (Fig. $2 L-$ $Q)$. Unexpectedly, line scans revealed transients of different amplitudes depending on where the SSR was scanned (Fig. $2 M-P$ ), presumably reflecting the "hot spots" of release previously visualized by others using postsynaptic $\mathrm{Ca}^{2+}$ reporters (Guerrero et al., 2005; Melom et al., 2013; Gratz et al., 2019). These hot spots also presumably underlie the large variance in $\mathrm{pH}$ transient peak values between NMJs. The average alkaline transient peaked at an amplitude of $1.22 \pm 0.45 \%(\mathrm{SD}, N=6)$, equivalent to a $\mathrm{pH}$ shift of $0.016 \log$ units (Fig. 2Q).

\section{Alkaline transients can be detected using chemical $\mathrm{pH}$ indicators targeted to the cleft}

To independently verify that we were detecting $\mathrm{pH}$ change in the synaptic cleft, we used a hybrid $\mathrm{pH}$ indicator that combines the subcellular targeting specificity of GEpHIs (Fig. 3A-C) with the intrinsic speed and brightness of chemical $\mathrm{pH}$ indicators. The hybrid, named PMCA-kisser-FAP, uses an extracellular fluorogenactivating peptide (FAP) that can bind a $\mathrm{pH}$-sensitive chemical (fluorogen). The intracellular domain of PMCA-kisser-FAP duplicates select elements of the Drosophila PMCA C terminus and localizes PMCA-kisser-FAP to the microenvironment of the PMCA (Fig. 3B) (Stawarski et al., 2018). Fluorogens are effectively nonfluorescent before binding a FAP tag. Acidification increases fluorescence of the fluorogen (se-Red-pH), whereas alkalinization reduces its fluorescence (Fig. 3C). PMCA-kisserFAP was expressed in muscles. The muscles were then exposed to membrane-impermeant se-Red-pH, resulting in a strong fluorescence signal in the SSR (Fig. 3D). se-Red-pH detected by the EMCCD camera reported rapid fluorescent transients in the synaptic cleft in response to single APs (Fig. $3 F$ ) with an amplitude $(-0.76 \pm 0.34 \%, \mathrm{SD}, N=6)$, time to peak $(77 \pm 27 \mathrm{~ms}, \mathrm{SD}, N=$ $6)$, and time course of decay ( $64 \pm 13 \mathrm{~ms}, \mathrm{SD}, N=6)$ indistinguishable from the $\mathrm{pH}$ transients reported by $\mathrm{pHusion}-\mathrm{Ex}$ in Figure $2 G(p=0.57, p=0.30$, and $p=0.26$, respectively; $\mathrm{df}=9$ for each, Student's $t$ tests). Subsequent ex vivo calibration of PMCA-kisser-FAP bound se-Red-pH (Fig. $3 E, F$ ) indicated that a single AP evokes an alkaline transient of $0.009(\Delta \mathrm{pH})$, similar to that reported by pHusion-Ex under the same conditions $(100 \mu \mathrm{M}$ ryanodine, $2 \mathrm{mM} \mathrm{Ca}^{2+}$ ). These results confirm that the phenomenon reported by pHusion-Ex was indeed a change in $\mathrm{pH}$, alkaline in polarity, rapid in onset, and short-lived.

\section{AP evoked cleft alkalinization decays like cytosolic $\mathrm{Ca}^{2+}$ transients}

If cleft alkaline transients are linked to the $\mathrm{Ca}^{2+} / \mathrm{H}^{+}$antiporting action of PMCA in the postsynaptic membrane, the postsynaptic cytosolic $\mathrm{Ca}^{2+}$ signals should be synchronous with cleft $\mathrm{pH}$ signals. To investigate this, we engineered a "coincidence" detector with a genetically encoded $\mathrm{Ca}^{2+}$ indicator (jRGECO1a) in the cytosol and a GEpHI (SE-pHluorin) in the cleft (Fig. 4A,B). When expressed in the muscle (Fig. $4 C-E$ ), it revealed that postsynaptic $\mathrm{Ca}^{2+}$ transients coincided with cleft $\mathrm{pH}$ transients and was subsequently deployed to establish the degree to which the two signals were correlated (Fig. $4 F-H$ ). Close examination of the SE-pHluorin signal (Fig. $4 I$ ) shows that it is not elevated by $50 \%$ until $\sim 40 \mathrm{~ms}$ after the nerve stimulus, and this had been previ- 
A
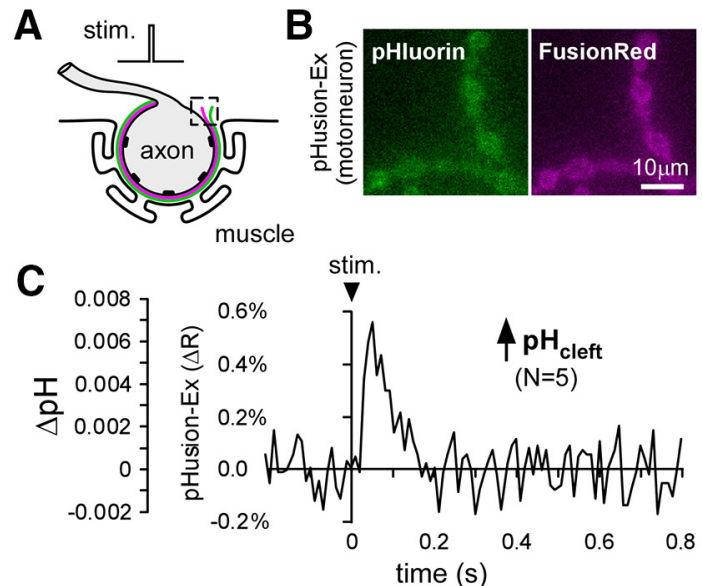

D
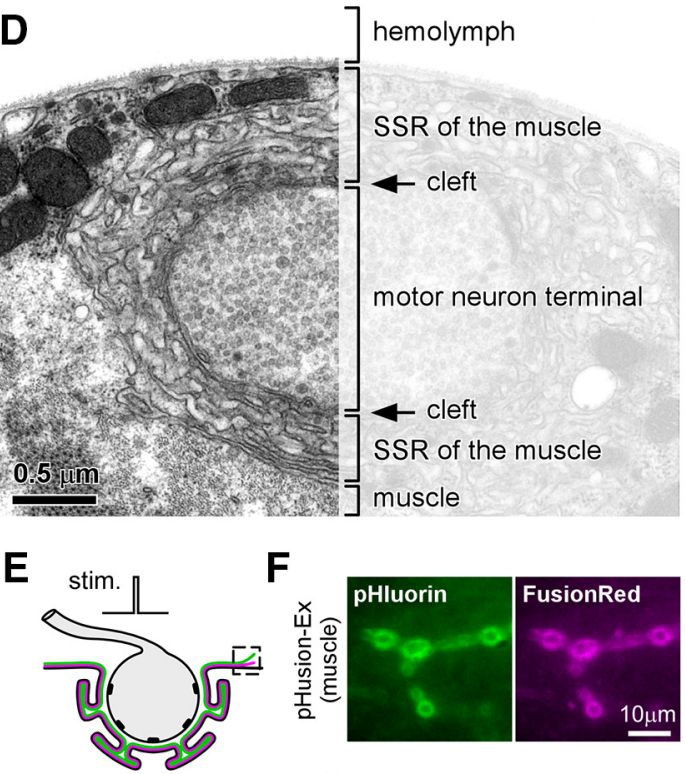

$F$

G
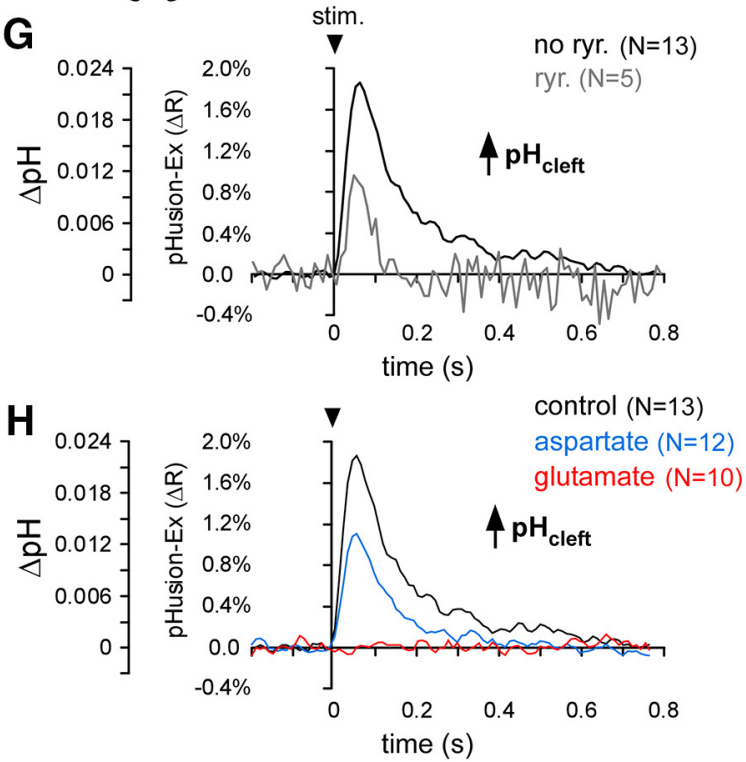

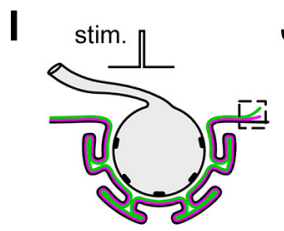

K
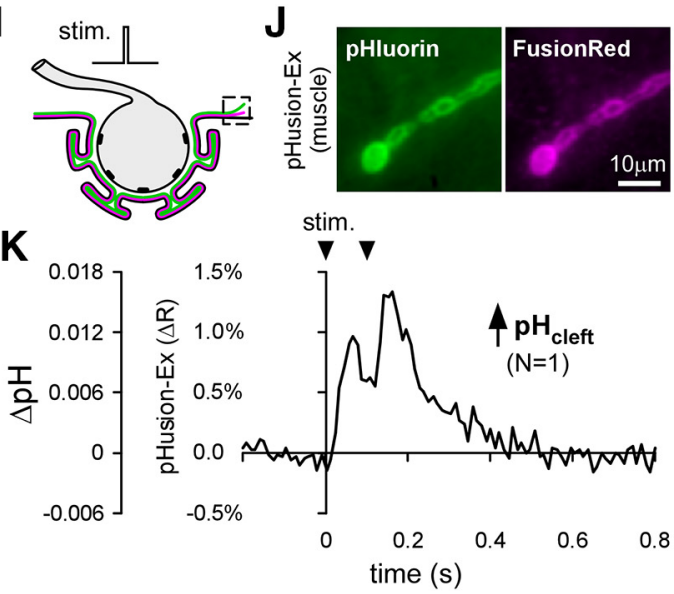

L
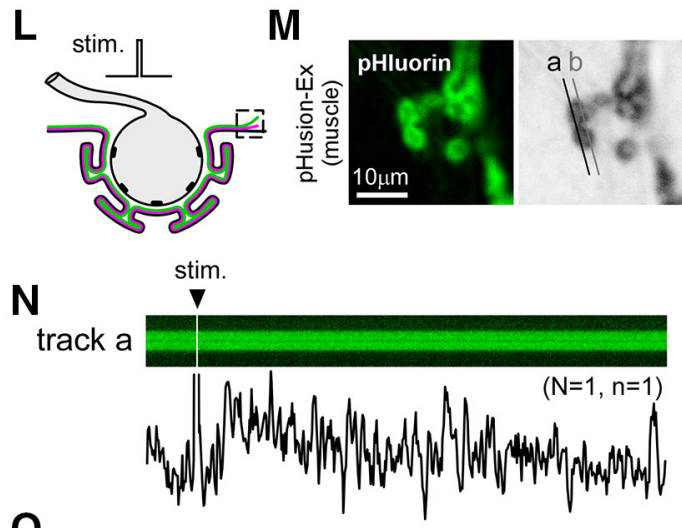

0
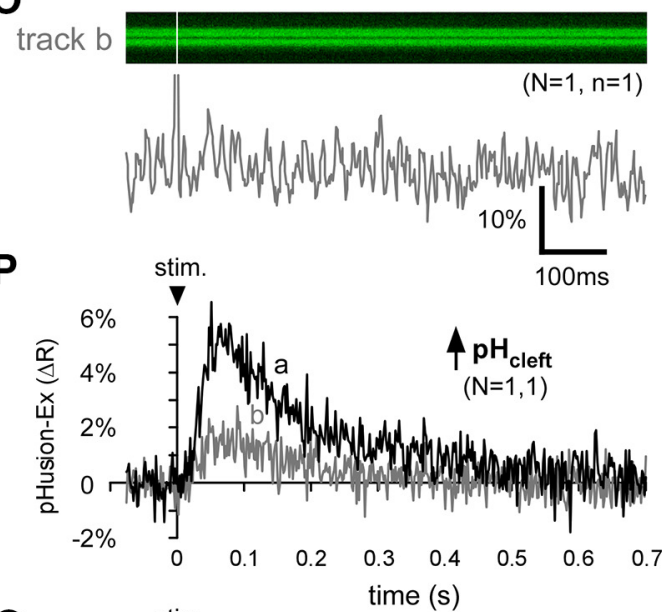

Q

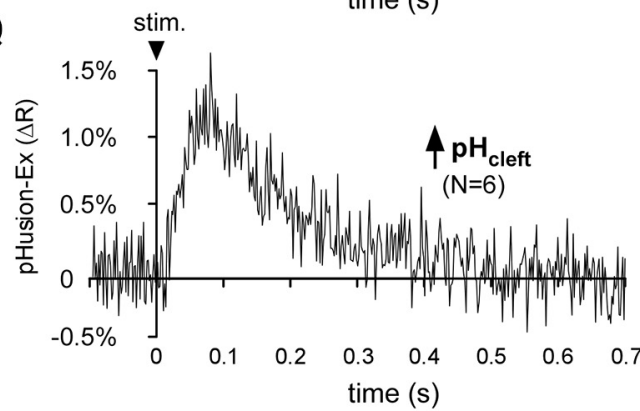

Figure 2. Imaging pHusion-Ex at the ex-vivo NMJ reveals alkalinization of the synaptic cleft in response to individual APs. $\boldsymbol{A}-\boldsymbol{K}$, Fluorescence data (ratios) were collected on an EMCCD camera at $94 \mathrm{~Hz}$. A, NMJ cross section, showing the cleft distribution of pHusion-Ex expressed using MN driver nSyb-GAL4. $\boldsymbol{B}$, Example images of both SE-pHluorin and FusionRed fluorescence under functional imaging conditions. $C$, pHusion-Ex ratio during isolated nerve stimuli: average of 100 stimuli $(n=100)$ delivered at $1 \mathrm{~Hz}$, at $5 \mathrm{NMJs}(\mathrm{N}=5)$. $\Delta \mathrm{pH}$ values, calculated from the change in the pHusion ratio, at far left (calibration shown in Fig. $1 E$ ). $D$, A transmission electron micrograph of a section through a $\mathrm{MN}$ terminal bouton (11,500 $\times$ magnification), embedded in the muscle which forms highly elaborated postsynaptic folds (SSR) around the bouton. $\boldsymbol{E}$, NMJ cross section showing the cleft distribution of pHusion-Ex expressed using muscle driver $24 \mathrm{~B}$-GAL4. $\boldsymbol{F}$, Example images of fluorescence from both fluorophores throughout the SSR. G, The pHusion-Ex ratio increased during nerve stimuli delivered at $1 \mathrm{~Hz}(n=100)$ in the presence or absence of (Figure legend continues.) 

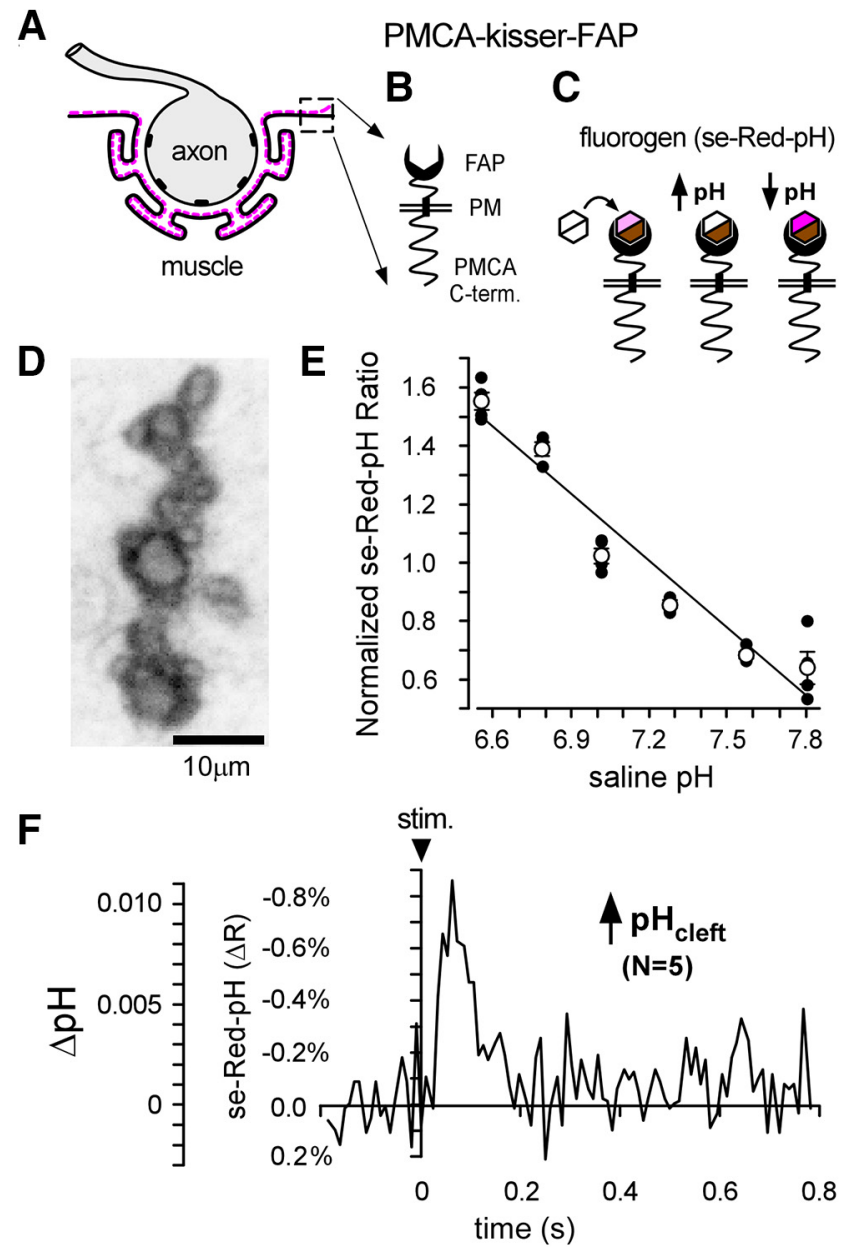

Figure 3. A chemical pH reporter trapped in the cleft at the ex vivo NMJ confirms the occurrence of AP-evoked alkaline transients. $A$, NMJ cross-section showing the cleft distribution of PMCA-kisser-FAP expressed using the muscle driver 24B-GAL4. B, Topology and composition of PMCA-kisser-FAP; a protein with an extracellular FAP fused to an intracellular engineered form of the Drosophila PMCA C terminus. Elements of the PMCA C terminus anchor it in the vicinity of endogenous PMCA at the NMJ (see Materials and Methods). C, The fluorogen, a PMimpermeable dye (se-Red-pH), has two absorbance maxima ( 551 and $633 \mathrm{~nm}$ ) and becomes fluorescent only upon binding to the FAP tag. Alkalinization results in a decrease in the fluorescence intensity ratio of se-Red-pH, whereas acidification leads to an increase in the ratio. $\boldsymbol{D}$, PMCA-kisser-FAP, labeled with se-Red-pH, localized to the SSR, and imaged live. E, Calibration curve (in situ) for PMCA-kisser-FAP bound se-Red-pH. Plotted values normalized to $\mathrm{pH}$ 7.2. $\boldsymbol{F}$, The average se-Red-pH fluorescence ratio during an isolated AP stimulus $(N=5, n=100$; impulses delivered at $1 \mathrm{~Hz}$ ) (left ordinate). Ratio measurements collected at $94 \mathrm{~Hz}$ on an EMCCD camera. Note the inverted ordinate. $\Delta \mathrm{pH}$ values, calculated from the change in the ratio, at far left, and reveal alkalinization of the synaptic cleft. $2 \mathrm{~mm} \mathrm{Ca}^{2+}, 20 \mathrm{~mm} \mathrm{Mg}^{2+}$, and $100 \mu \mathrm{M}$ ryanodine used throughout.

$\leftarrow$

(Figure legend continued.) $\quad$ ryanodine. $\boldsymbol{H}$, Extracellular glutamate at $7 \mathrm{~mm}$ occludes alkalinization of the synaptic cleft, whereas $7 \mathrm{~mm}$ aspartate does not. I, NMJ cross section showing cleft localization of muscle expressed (24B-GAL4) pHusion-Ex. J, Example images of fluorescence in the SSR, as in $\boldsymbol{F}$. $\boldsymbol{K}$, The pHusion-Ex ratio increased substantially in response to two stimuli delivered with an interval of $100 \mathrm{~ms}$ (average of 100 paired stimuli delivered at $1 \mathrm{~Hz}$ ). $\mathbf{L}-\mathbf{Q}$, Fluorescence data were collected on a CLSM at $560 \mathrm{~Hz}$. L, NMJ cross section showing muscle localization of pHusion-Ex. M, Left, CLSM image of SE-pHluorin fluorescence in the SSR. Right, Annotation to show the tracks of two different scans (a and b). $\boldsymbol{N}$, Top, A series of lines scanned along " $a$ " in $\boldsymbol{M}$. The time of nerve impulse delivery is coincident with a white vertical LED scar. Bottom, Fluorescence intensity plot of the scanned lines shown as $\% \Delta F / F\left(\left[\left(F_{\text {stim }}-F_{\text {rest }}\right) / F_{\text {rest }}\right]\right.$ $\times 100)$. $\boldsymbol{O}$, Same as in $\boldsymbol{N}$, but for a series of lines scanned along " $b$ " in $\boldsymbol{M}$. $\boldsymbol{P}$, Plots of the average responses along each track $(N=1, n=100$; impulses delivered at $1 \mathrm{~Hz})$. $\mathbf{Q}, \mathbf{A}$ plot of the average response to nerve impulses delivered at $1 \mathrm{~Hz}$, from $6 \mathrm{NMJs}(N=6)(n=100$ each). 2 $\mathrm{mm} \mathrm{Ca}{ }^{2+}$ and $20 \mathrm{~mm} \mathrm{Mg}^{2+}$, used throughout. $C, G, K, 100 \mu \mathrm{m}$ ryanodine used to stifle muscle contraction. ously observed when pHusion-Ex alone was expressed presynaptically and examined by EMCCD camera in the presence of ryanodine ( $39.4 \pm 4.7 \mathrm{~ms}, N=5$; e.g., Fig. $2 C$ ), or postsynaptically ( $40.3 \pm 6.0 \mathrm{~ms}, N=5$; Fig. $2 G$ ). GECIs have much slower kinetics than most chemical $\mathrm{Ca}^{2+}$ indicators (Pérez Koldenkova and Nagai, 2013), and so it is possible that the jRGECOla $\mathrm{Ca}^{2+}$ signal is misleadingly slow. The change in SE-pHluorin fluorescence, however, relies on direct protonation of a chromophore (Miesenböck et al., 1998) and thus should reflect $\mathrm{pH}$ change with good temporal fidelity. Certainly, the EMCCD camera and confocal laser scanning microscopy (CLSM) are capable of reporting transients with a rapid onset, and they report presynaptic $\mathrm{Ca}^{2+}$ elevation without delay when imaging fluorescent chemical $\mathrm{Ca}^{2+}$ indicators in presynaptic terminals: EMCCD (Lu et al., 2016) and CLSM (Macleod et al., 2002). In sum, the correlation of the cleft $\mathrm{pH}$ signal with the postsynaptic $\mathrm{Ca}^{2+}$ signal (Fig. $4 \mathrm{H}, \mathrm{I}$ ), the delay in cleft alkalinization relative to the presynaptic $\mathrm{Ca}^{2+}$ signal, and the occlusion of cleft alkalinization by occlusion of postsynaptic $\mathrm{Ca}^{2+}$ entry with glutamate (Fig. $2 \mathrm{H}$ ) all point to postsynaptic (rather than presynaptic) PMCA activity being responsible for cleft alkalinization.

\section{Burst-evoked cleft alkalinization is greatly susceptible to extracellular $\mathrm{pH}$ buffering capacity}

To examine the impact of extracellular $\mathrm{pH}$ buffering on the magnitude of cleft $\mathrm{pH}$ transients that arise during neurotransmission, we compromised the capacity of the bicarbonate-based buffering system, or increased buffering capacity through the addition of a zwitterionic buffer (HEPES). The change in cleft $\mathrm{pH}$ was monitored using postsynaptically expressed pHusion-Ex (Fig. 5A). Sodium bicarbonate $\left(\mathrm{NaHCO}_{3} ; 15 \mathrm{~mm}\right)$ was added to all solutions as bicarbonate-based buffering, catalyzed by endogenous CAs, is a staple for maintaining acid-base balance in both vertebrate and invertebrate systems. The nerve leading to the terminal boutons of MN6/7-Ib was stimulated at its average firing frequency (21 $\mathrm{Hz}$ ) (Chouhan et al., 2010) for the duration of a single motor pattern burst ( $\sim 950 \mathrm{~ms})$ (Klose et al., 2005), and this evoked modest alkalinization of the cleft (Fig. $5 B, C$, black trace; $\Delta \mathrm{R}$ $10.5 \pm 1.0 \%)$. However, if the saline was not continually bubbled with $\mathrm{CO}_{2}$, and allowed to lose bicarbonate $\left(\mathrm{HCO}_{3}^{-}\right)$, nerve stimulation evoked $261 \%$ greater alkalinization of the cleft (data not shown). Consistent with a role for bicarbonatebased buffering the addition of the CA blocker acetazolamide to the bubbled saline resulted in $69 \%$ greater alkalinization (Fig. $5 B, C$, blue trace; $\Delta \mathrm{R} 17.7 \pm 1.6 \%$ ), generally consistent with a low concentration of extracellular $\mathrm{CA}$ in the cleft (Chesler, 2003; Tong et al., 2006; Highstein et al., 2014). HEPES, which has a greater immediate buffering capacity than bicarbonate, reduced alkalinization by $52 \%$ when added at 5 $\mathrm{mm}$ to a bubbled saline already containing bicarbonate (Fig. $5 B, C$, red trace; $\Delta \mathrm{R} 5.0 \pm 0.6 \%$ ). The responses in control, acetazolamide, and HEPES were statistically different from one another (one-way ANOVA, multiple comparisons vs control, Holm-Sidak method; $\left.F_{(2,18)}=30.5, p<0.001\right)$. Despite the motor axons being driven at physiological rates, and only for a period equivalent to a single body-wall contraction during peristaltic locomotion, the summation of cleft $\mathrm{pH}$ transients was substantial. Nevertheless, the amplitudes of these alkaline transients ex vivo are well below those observed in vivo (Fig. $1 D, H$ ), indicating that the $\mathrm{pH}$ buffering capacity ex vivo is more effective than that in vivo, or, that our stimulus protocols are less intense than those occurring in an intact but restrained larva. 

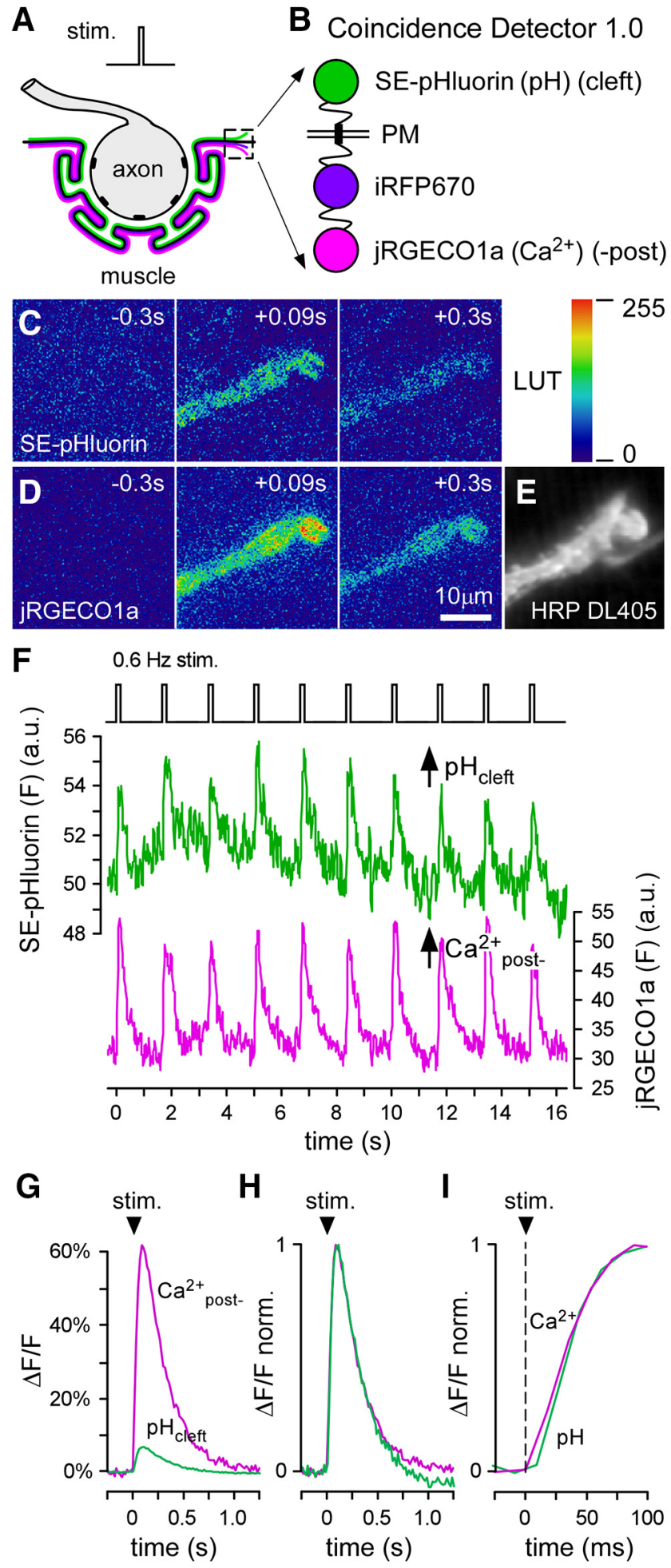

Figure 4. Cleft alkalinization is synchronous with $\mathrm{Ca}^{2+}$ extrusion from adjacent synaptic compartments. $A$, NMJ cross-section, showing the distribution of coincidence detector 1.0 construct expressed using the muscle driver 24B-GAL4. B, The topology and composition of the construct relative to the cleft. C, D, Images of coincidence detector 1.0 at a live NMJ (MN6/7-lb): (C) SE-pHluorin fluorescence and (D) jRGEC01a fluorescence imaged $0.3 \mathrm{~s}$ before, $0.09 \mathrm{~s}$ after, and $0.3 \mathrm{~s}$ after a nerve stimulus. Each image represents an $8 \mathrm{~ms}$ exposure averaged from 50 trials (50 stimuli delivered at $0.6 \mathrm{~Hz}$ ). The look-up-table (LUT) used in $\boldsymbol{C}$ and $\boldsymbol{D}$ is shown in $\boldsymbol{C}$. $\boldsymbol{E}$, Fluorescence image from an HRP antibody conjugated with DyLight 405 at the live NMJ. $F$, Plots represent the fluorescence intensity of SE-pHluorin and jRGEC 01 a at the terminals in $\mathbf{C}$ and $\boldsymbol{D}$, minus background, at each sampling interval over 10 stimuli (fluorescence from each fluorophore was collected on the EMCCD camera at $56 \mathrm{~Hz}$ and interdigitated with the other fluorophore). $\mathbf{G}$, Average response $\left(\% \Delta \mathrm{F} / \mathrm{F}_{\text {rest }}\right)$ of each fluorophore to a single nerve stimulus
The cleft of the mouse calyx of Held synapse alkalinizes during activity

Individual AZs of the calyx of Held synapse show the architecture of conventional neuronal synapses, and previous electrophysiological and $\mathrm{Ca}^{2+}$ imaging data from the mouse indicate that the cleft acidifies due to corelease of glutamate and protons (González-Inchauspe et al., 2017). However, no pH imaging data are available. The calyx of Held synapse is a giant axosomatic synapse in the auditory brainstem (Fig. 6A) (Borst and Soria van Hoeve, 2012), and, like the Drosophila NMJ, the cleft is spatially restricted at AZs but can form extended extracellular spaces away from AZs (Fig. 6B-D) (Rowland et al., 2000). Adeno-associated viral vectors encoding $\mathrm{pHusion}-\mathrm{Ex}$ constructs were injected into the ventral cochlear nucleus at $\mathrm{P} 1$, and by $\sim \mathrm{P} 21$ sparsely labeled calyces could be seen in the contralateral medial nucleus of the trapezoid body (Fig. $6 E, F$ ). Imaging of individual calyces in slices during perfusion with bicarbonate/HEPES ACSF buffered to different $\mathrm{pH}$ (see Materials and Methods) demonstrated the extracellular localization of pHusion-Ex and its ability to report $\mathrm{pH}$ in a linear range around neutral (Fig. $6 G$ ), as observed during ex vivo calibrations in Drosophila (Fig. $1 E$ ). In response to a short burst of APs (1 s, $400 \mathrm{~Hz}$ ), pHusion-Ex revealed immediate cleft alkalinization through a small but significant increase in its ratio relative to baseline ( $\Delta \mathrm{R}: 0.93 \pm 0.22 \% \mathrm{SEM} ; \Delta \mathrm{pH}: \sim 0.01 ; N=9 ; t=$ $5.843, \mathrm{df}=8, p=0.0004$, one-sample $t$ test vs 0 ; Fig. $6 H$ ), which was diminished in the presence of $5 \mathrm{~mm} \operatorname{HEPES}(t=4.306, \mathrm{df}=$ $7, p=0.0035$, paired Student's $t$ test; Fig. $6 I$ ). Consistent with cleft alklinization being the product of acid movement across synaptic membranes, pHusion expressed freely in the cytosol (cyto-pHusion) of the calyx (Fig. $6 \mathrm{~J}, \mathrm{~K}$ ) revealed presynaptic acidification in response to the same burst of activity $(\Delta \mathrm{R}: 2.82 \pm$ $0.61 \%$ SEM; $\Delta \mathrm{pH}: \sim 0.03 ; N=7)$, which was significant relative to baseline ( $t=4.685$, df $=6, p=0.0034$, one-sample Student's $t$ test; Fig. $6 \mathrm{M}, N)$. As predicted from its location, acidification reported by intracellular cyto-pHusion was refractory to the presence of $5 \mathrm{mM}$ extracellular HEPES $(t=0.085$, $\mathrm{df}=3, N=4$, $p=0.9379$, paired Student's $t$ test; Fig. $6 N)$. Primary cortical neurons transduced with the same vectors also demonstrated appropriate cytosol versus plasma membrane targeting of cytopHusion and pHusion-Ex constructs, respectively (data not shown). In sum, these data demonstrate that alkalinization also occurs at some central mammalian glutamatergic synapses during activity.

\section{A GEpHI that localizes with $\mathrm{Ca}^{2+}$ channels within the cleft confirms alkalinization at AZs}

The extracellular $\mathrm{pH}$ changes reported in the preceding figures may be dominated by GEpHIs not within the AZs proper. The GEpHIs have been alternately targeted to presynaptic and postsynaptic membranes, but the cumulative area of the SSR of the Drosophila NMJ and the extended extracellular spaces of the mouse calyx of Held will far exceed the cumulative area of the AZs. This leaves open the possibility that the AZ proper acidifies as acid is released from SVs, but the rest of the cleft alkalinizes, resulting in a net GEpHI signal reporting alkalinization. To investigate this possibility, we targeted a GEpHI to the AZ proper

$\leftarrow$

(data from $\boldsymbol{C}$ and $\boldsymbol{D}$, i.e., all 50 stimuli), where $\mathrm{F}_{\text {rest }}$ is the average fluorescence value immediately before each nerve stimulus. $\boldsymbol{H}$, Data from $\boldsymbol{G}$ normalized to a peak value of 1. I, Data from $\boldsymbol{H}$ plotted on an expanded time scale at the time of nerve stimulus. $2 \mathrm{~mm} \mathrm{Ca}^{2+}, 20 \mathrm{~mm} \mathrm{Mg}^{2+}$, and $100 \mu \mathrm{m}$ ryanodine used throughout. 
A

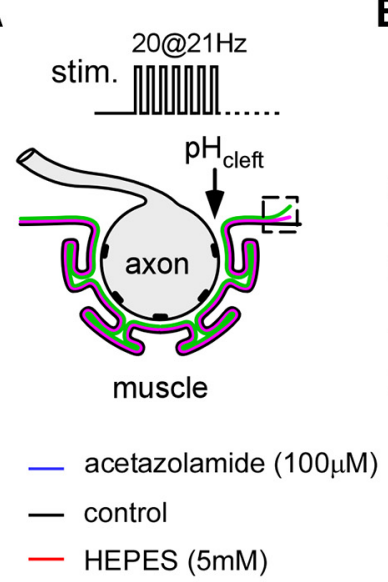

B

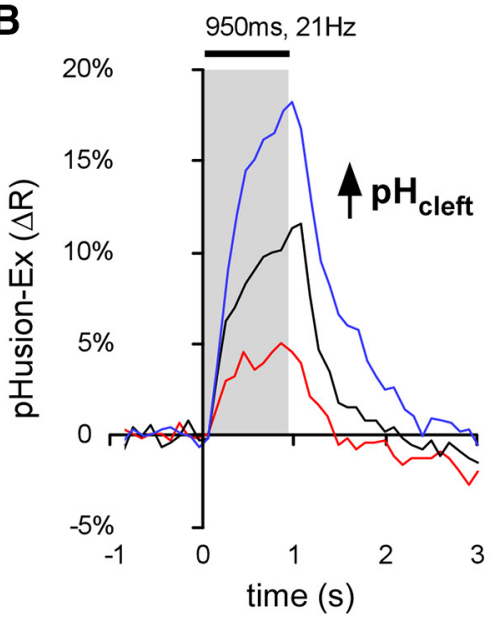

C

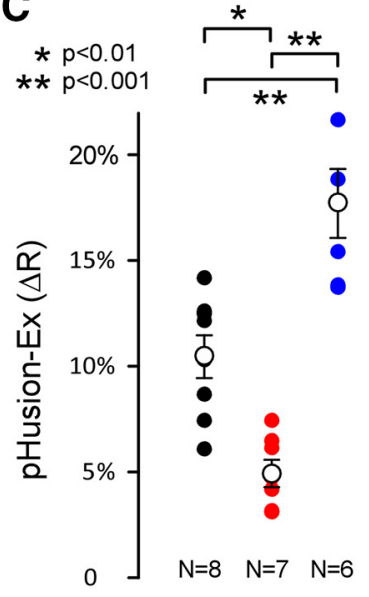

Figure 5. Burst-evoked cleft alkalinization is modulated by extracellular pH buffering capacity. $A$, NMJ cross section, showing the cleft distribution of pHusion-Ex expressed in muscle (24B-GAL4). $B$, Averaged pHusion-Ex ratio responses to $21 \mathrm{~Hz}$ trains $\left(950 \mathrm{~ms}\right.$ ) of impulses delivered to the nerve under different extracellular buffering conditions. Black trace represents $15 \mathrm{~mm} \mathrm{NaHCO}_{3}(\mathrm{~N}=8)$. Blue trace represents $15 \mathrm{~mm} \mathrm{NaHCO}$ with $100 \mu$ m acetazolamide added to block CA ( $N=6)$. Red trace represents $10 \mathrm{~mm} \mathrm{NaHCO}{ }_{3}$, with $5 \mathrm{~mm} \mathrm{HEPES}(\mathrm{N}=7)$. Ratio measurements collected at $10 \mathrm{~Hz}$. $C, A$ plot of the pHusion-Ex peak change in ratio $(\Delta R)$ in individual experiments. Muscle contractions were blocked through 30 min prior incubation in $100 \mu \mathrm{m}$ ryanodine.

(Fig. 7) where the cleft is only $\sim 20$ nm wide in Drosophila (Pawlu et al., 2004) similar to synaptic clefts within the mouse brain (Peters et al., 1991). As described previously (Stawarski et al., 2018), Cac-kisser probes were designed to localize to the Drosophila presynaptic VGCC (Cacophony). In this case, Cac-kisserpHerry-TM is comprised of an intracellular carboxy terminus based on Cac, a TM of hPDGFR, and SE-pHluorin fused to mCherry exposed to the extracellular space (Fig. 7A). When expressed in MNs, the presynaptic fluorophores give a punctate appearance (Fig. $7 B$ ). This punctate distribution contrasts with a more robust signal from pHerry-Ex expressed in MNs, which also exposes a fusion of SE-pHluorin and mCherry to the extracellular space (Fig. $7 C, D$ ). In response to a short burst of APs, Cac-kisserpHerry-TM revealed immediate cleft alkalinization through an increase in its ratio $(\Delta \mathrm{R}: 25.8 \pm 9.1 \% \mathrm{SD} ; \Delta \mathrm{pH}: \sim 0.32 ; N=6$; Fig. $7 E, F)$. As the signal from this restricted area of the presynaptic membrane is very weak, a stimulus frequency of twice the endogenous frequency was used $(42 \mathrm{~Hz})$, and the saline was allowed to deplete of $\mathrm{HCO}_{3}^{-}$. In contrast, pHerry-Ex, which was not confined to the $\mathrm{AZ}$ proper, yielded a smaller response under the same conditions $(\Delta \mathrm{R}: 14.9 \pm 5.4 \% \mathrm{SD} ; \Delta \mathrm{pH}: \sim 0.19 ; N=8$; $t=2.936, \mathrm{df}=12, p=0.012$, Student's $t$ test; Fig. $7 E, F)$. In a separate experiment, under the same conditions, a $\mathrm{Ca}^{2+}$ indicator expressed in the muscle reveals a large $\mathrm{Ca}^{2+}$ signal evoked in the SSR (Fig. 7E). Confocal microscopic examination of both live (Fig. 7G) and fixed (Fig. 7H) NMJs reveals Cac-kisserpHerry-TM to show the characteristic punctate pattern of presynaptic Cacophony (Gratz et al., 2019), which localizes with punctae of the AZ protein Bruchpilot (Brp). It is not clear why the AZ proper should become more alkaline, as the volume of the extended cleft might be expected to equilibrate during trains of APs, but it is clear that the AZ proper does not acidify.

\section{Discussion}

Whether neurotransmission drives cleft acidification at conventional neuronal synapses is of paramount interest, as implications follow for STSP depending on the polarity, magnitude, and kinetics of any $\mathrm{pH}$ transients. Here, we tested the hypothesis that neuronal synapses with non-ribbon-type AZs acidify during neurotransmission by targeting $\mathrm{pH}$ indicators to the outer leaflet of the presynaptic or postsynaptic plasma membrane, adjacent to the PMCA or adjacent to VGCCs. In every instance, the cleft was found to alkalinize in response to nerve stimulation. Cleft alkalinization is not a unique feature of the Drosophila NMJ as the same $\mathrm{pH}$ indicators targeted to the mouse calyx of Held synapse revealed cleft alkalinization during burst firing. Cleft alkalinization is consistent with $\mathrm{Ca}^{2+}$ clearance from adjacent compartments via the PMCA, which antiports $\mathrm{H}^{+}$for $\mathrm{Ca}^{2+}$. If indeed the PMCA does drive cleft alkalinization, then cleft alkalinization likely occurs at all synapses that use the PMCA to clear $\mathrm{Ca}^{2+}$, and these comprise a large subset of mammalian central synapses (Brini et al., 2017). As the Drosophila NMJ and mouse calyx of Held synapses are relay synapses, they might be considered no more analogous to "conventional" mammalian central synapses than ribbon-type synapses. However, similarity to conventional neuronal synapses can be found at the level of AZs, where the average probability of SV release in response to an AP is low at both the Drosophila NMJ (0.11-0.33) (Lu et al., 2016; Newman et al., 2017) and rat calyx (0.25-0.4) (Meyer et al., 2001), which contrasts greatly with the tens of SVs that might be released within several milliseconds at a ribbon synapse (von Gersdorff et al., 1996; DeVries, 2001).

There is little doubt that clefts of ribbon synapses acidify during activity, as this has been revealed through direct imaging of SE-pHluorin fused to an extracellular $\mathrm{Ca}^{2+}$-channel subunit $\left(\alpha_{2} \delta_{4}\right)$, which placed SE-pHluorin within the cleft adjacent to AZs (Wang et al., 2014; Beckwith-Cohen et al., 2019). Their approach was approximated in this study where a "kisser" probe (Stawarski et al., 2018) placed SE-pHluorin and mCherry in the cleft adjacent to presynaptic VGCCs (Fig. $7 A, B$ ). Rather than acidification, we observed alkalinization following activity (Fig. $7 E, F)$. The difference in polarity of cleft $\mathrm{pH}$ signals between the studies might be attributed to a higher rate of SV exocytosis at AZs in ribbon synapses (DeVries, 2001; Cho and von Gersdorff, 2014). At retinal ribbon synapses, cleft acidification has also been attributed to vacuolar-type ATPase-mediated $\mathrm{H}^{+}$efflux from depolarizing postsynaptic cells (horizontal cells) (Hirasawa et al., 2012; Wang et al., 2014). Cone photoreceptor clefts acidify by $\sim 0.14 \mathrm{pH}$ units as neurotransmission recommences subsequent to a light flash (Wang et al., 2014), whereas Drosophila NMJ clefts alkalinize by $\sim 0.12 \mathrm{pH}$ units during a physiological-grade stim- 


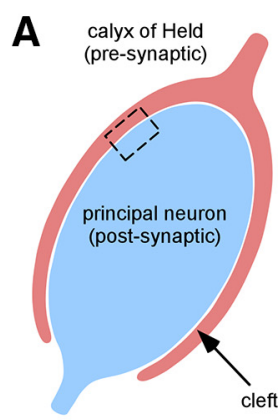

E

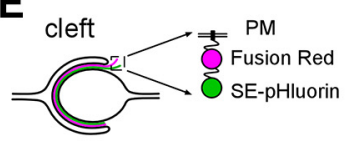

F

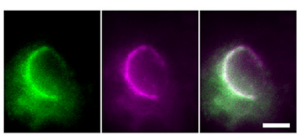

J

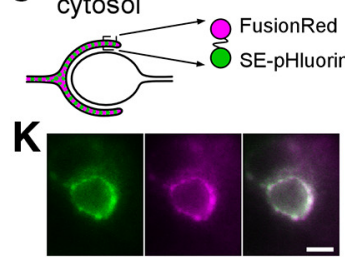

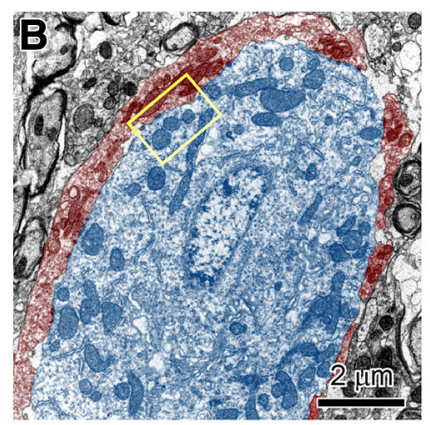

G

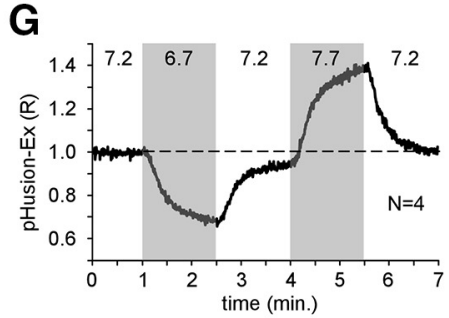

L

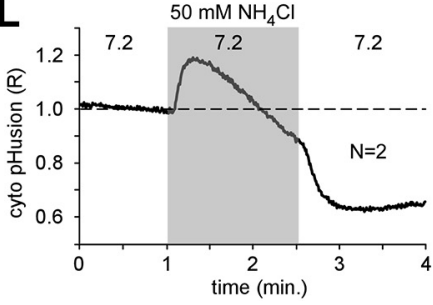

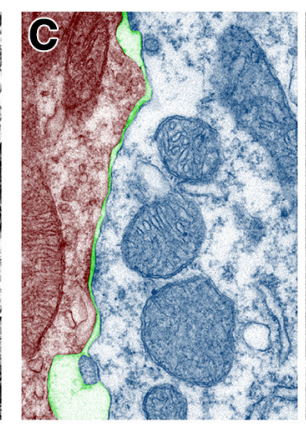

H

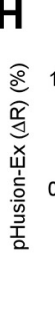

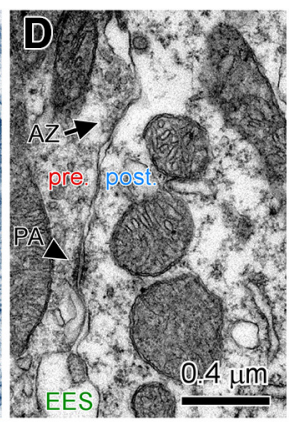

I
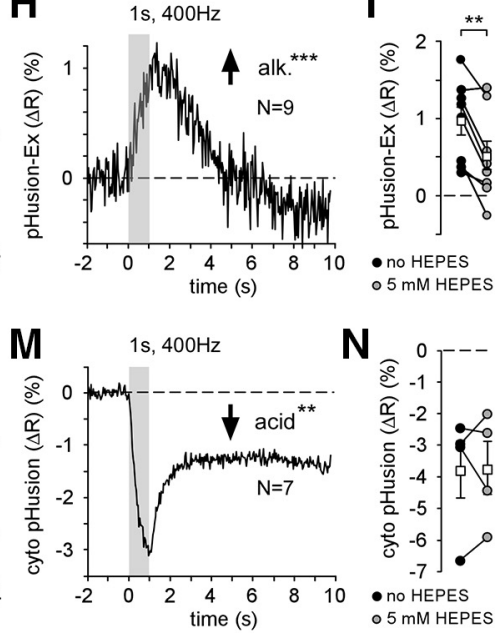

N

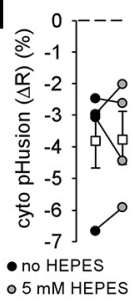

Figure 6. Activity-dependent pH changes at the mammalian calyx of Held synapse. A, Representation of the calyx of Held synapse, highlighting the presynaptic terminal (red), postsynaptic cell body (blue), and synaptic cleft between the presynaptic and postsynaptic compartments. $\boldsymbol{B}$, Transmission electron micrograph illustrating the synapse from a P22 mouse. False-color is overlaid using the color code in $\boldsymbol{A}$. Note extended apposition of presynaptic (red) and postsynaptic (blue) membranes. $\boldsymbol{C}$, Magnification of the boxed area marked in $\boldsymbol{B}$, with false-color highlighting presynaptic terminal (red), postsynaptic cell (blue), and synaptic cleft (green). $\boldsymbol{D}$, An unobscured view of the section shown in C. Presynaptic AZ is identified as presynaptic electron-dense material surrounded by SVs. Trans-synaptic puncta adherentia (PA) are also observed as dense material on opposing membranes, but lack SV clusters. An example of extended extracellular spaces is also captured in this image and is characteristic of these synapses. $\boldsymbol{E}$, Schematic representation of expression pattern of pHusion-Ex probe. Fluorophores are anchored to the calyx of Held presynaptic membrane and exposed to the extracellular synaptic cleft milieu (as well as outside the cleft). $\boldsymbol{F}$, Wide-field fluorescence image of pHusion-Ex expression at the synapse in a brain slice, showing spatially restricted expression at the presynaptic terminal, and colocalization of the two fluorophores. Left, SE-pHluorin (green). Middle, FusionRed (magenta). Right, Merged image. Scale bar, $10 \mu \mathrm{m}$. G, Average response of $N=4$ pHusion-Ex-expressing terminals to changes in extracellular $\mathrm{pH}$. Bath pH indicated at top of plot. pHusion-Ex was bidirectionally responsive to decreased (6.7) and increased (7.7) bath $\mathrm{pH}$. $\boldsymbol{H}$, Cleft pH increases during synaptic activity. Plot of average pHusion-Ex ratio (divided by the initial pHusion-Ex ratio) during high-frequency stimulation ( $400 \mathrm{~Hz}, 1 \mathrm{~s}$; gray bar). Note the clear increase in $\mathrm{pH}$ in the synaptic cleft during activity. ${ }^{* * *} p=0.0004$. I, Summary plot of pHusion-Ex responses to activity in low (no HEPES) and high (5 mm HEPES) pH buffering media. Filled symbols represent individual peak pHusion-Ex responses. Open squares represent mean \pm SEM. ${ }^{* *} p=0.0035$. J, Representation of cyto-pHusion probe at the calyx of Held synapse, where the probe is restricted to the presynaptic cytosol. $\boldsymbol{K}$, Example wide-field fluorescence image of cyto-pHusion showing specific presynaptic localization. Left, SE-pHluorin (green). Middle, FusionRed (magenta). Right, Merged image. Scale bar, $10 \mu \mathrm{m}$. L, Average response of $\mathrm{N}=2$ cyto-pHusion-expressing terminals to bath-applied $50 \mathrm{~mm} \mathrm{NH}_{4} \mathrm{Cl}$ in a saline buffered to 7.2 . 0 n exposure to $\mathrm{NH}_{4} \mathrm{Cl}$, initial alkalinization by $\mathrm{NH}_{3}$ entry is followed by plateau-phase acidification due to $\mathrm{NH}_{4}{ }^{+}$accumulation in the cytosol. $M$, Synaptic activity acidifies the presynaptic cytosol. Plot $(N=7)$ of average cyto-pHusion ratio (divided by the initial cyto-pHusion ratio) during high-frequency stimulation $(400 \mathrm{~Hz}, 1 \mathrm{~s})$. The terminal rapidly acidifies during activity $(* * p=0.0034)$, and rapidly but partially recovers after stimulation is terminated. $\boldsymbol{N}$, Summary plot of cyto-pHusion responses unaffected by the level of extracellular pH buffering. Filled symbols represent individual cyto-pHusion peak responses. Open squares represent mean \pm SEM.

ulus train (Fig. $5 B, C$ ). Ultimately, a direct comparison of magnitudes provides limited insight if different mechanisms underlie the $\mathrm{pH}$ transients. A nonvesicular source of protons (e.g., from depolarizing postsynaptic cells) may explain the slow time course of cleft acidification at the retinal ribbon synapses (tau $\sim$ several hundred milliseconds), yet it is not so different from the progressive cleft alkalinization during a train at the Drosophila NMJ (Fig. $5 B)$.

Release at retinal ribbon synapses is mediated by graded depolarization, whereas release at an NMJ is triggered by an AP. The tight synchronicity at the NMJ provides an ideal opportunity to temporally characterize cleft $\mathrm{pH}$ consequences of neurotransmission. Following a single nerve impulse, the time course of cleft alkalinization and reacidification is in close register with the postsynaptic cytosolic $\mathrm{Ca}^{2+}$ signal measured with a GECI (Fig. 4G-I). Indeed, electroneutral exchange of $\mathrm{Ca}^{2+}$ for $2 \mathrm{H}^{+}$across the
PMCA has been shown to alkalinize the extracellular space in different portions of mammalian hippocampal and cerebellar cortical preparations (for review, see Chesler, 2003; Makani and Chesler, 2010a). The PMCA plays a prominent role in $\mathrm{Ca}^{2+}$ extrusion from both presynaptic and postsynaptic compartments at the Drosophila NMJ (Lnenicka et al., 2006; Desai and Lnenicka, 2011), and the data presented here are consistent with the PMCA generating the activity-dependent alkaline transients. For example, the reduced magnitude of alkalinization at the calyx synapse relative to the Drosophila larval NMJ is consistent with a reduced requirement for PMCA extrusion. At the calyx of Held, only $\sim 25 \%$ of presynaptic $\mathrm{Ca}^{2+}$ influx is extruded via the PMCA (Kim et al., 2005), and activity-dependent postsynaptic $\mathrm{Ca}^{2+} \mathrm{en-}$ try is limited (Lujan et al., 2019). While our data are fully in support of the PMCA as the mechanism underlying cleft alkalinization, further experiments may be required for confirmation. 
A
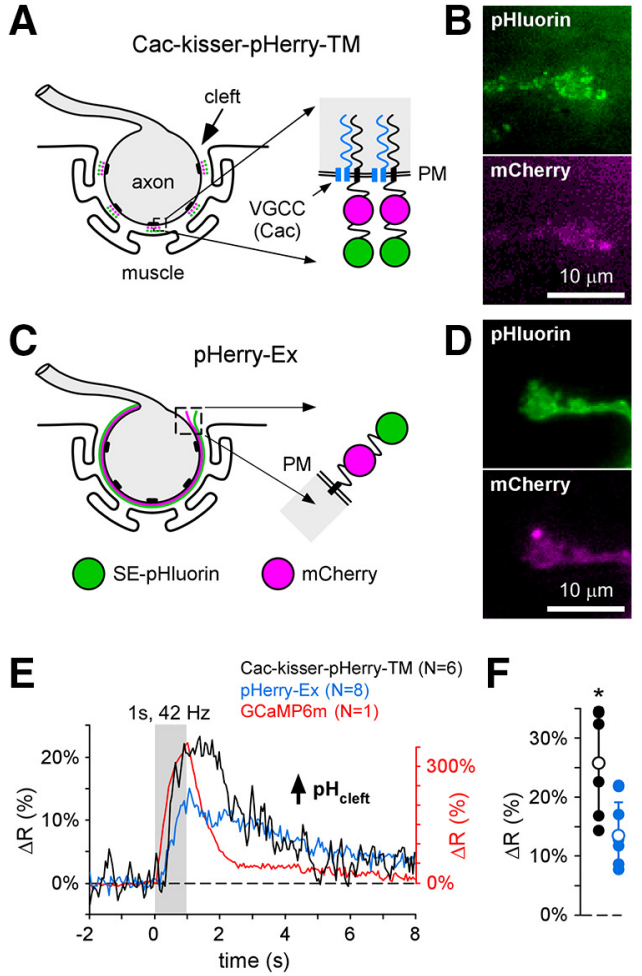

G Cac-kisser-pHerry-TM (live)

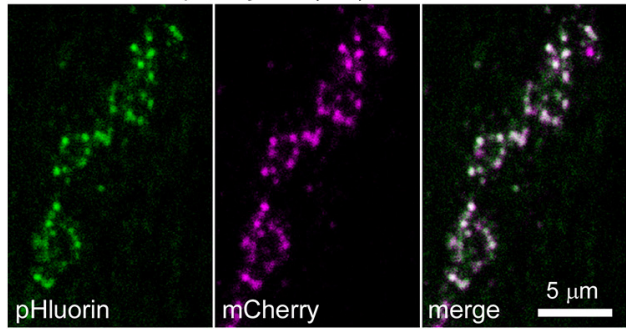

H Cac-kisser-pHerry-TM (fixed)

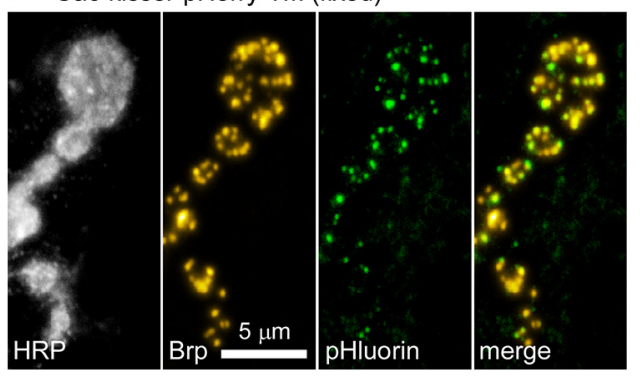

Figure 7. Imaging Cac-kisser-pHerry-TM at the ex vivo NMJ confirms alkalinization within the cleft at the AZ proper. $A$, Topology and composition of Cac-kisser-pHerry-TM at the Drosophila NMJ. SE-pHluorin is fused to mCherry in the extracellular space while an intracellular engineered form of the Drosophila $C_{\mathrm{v}} 2$ VGCCs (Cacophony, $\mathrm{Cac}$ ) C terminus localizes the fluorophores laterally within the cleft at the $\mathrm{AZ} \mathrm{Ca}^{2+}$ channels. $\boldsymbol{B}$, Example images of presynaptic SE-pHluorin and mCherry fluorescence of Cac-kisser-pHerry-TM under functional imaging conditions. C, Topology and composition of pHerry-Ex; similar to pHusion-Ex (Fig. 1C). D, Example images of presynaptic SE-pHluorin and mCherry fluorescence of pHerry-Ex. Presynaptic expression driven by MN driver $\mathrm{Syb}$-GAL4 in $\boldsymbol{B}$ and $\boldsymbol{D}$. $\boldsymbol{E}$, The average ratio change in Cac-kisserpHerry-TM (cleft), pHerry-Ex (cleft), and GCaMP6m (postsynaptic) fluorescence $(\Delta R)$, in response to nerve stimulation ( 40 impulses delivered at $42 \mathrm{~Hz}$ ) at time $=0$. Data were collected from 6, 8, and 1 separate NMJs, respectively. Postsynaptic expression of myr-FusionRedGCaMP6m driven by 24B-GAL4. $\Delta \mathrm{R}(\%)$ shown for the $\mathrm{pH}$-sensitive probes on the left ordinate, $\mathrm{Ca}^{2+}$-sensitive probe on the right. Fluorescence data (ratios) were collected on an EMCCD camera at $19 \mathrm{~Hz}$. F, Plot of the stimulated ratio response from individual preparations. Data are mean $\pm S D .{ }^{*} p=0.012$ (Student's $t$ test). Muscle contractions were blocked through $30 \mathrm{~min}$ incubation in $100 \mu \mathrm{m}$ ryanodine. $\mathbf{G}$, Distribution of SE-pHluorin and mCherry of presynaptic
Alkalinization is not caused by neurotransmitter release per se, as $\mathrm{Ca}^{2+}$ in the extracellular space will allow exocytosis, but no $\mathrm{pH}$ cleft transient was detected if glutamate blocks $\mathrm{Ca}^{2+}$ permeation of postsynaptic glutamate receptors (Fig. $2 H$ ). This result emphasizes $\mathrm{Ca}^{2+} / \mathrm{H}^{+}$exchange across the postsynaptic membrane as the most substantial contributor to transient cleft alkalinization. Significantly, the absence of cleft alkalinization, even when presynaptic PMCA is active, does not preclude PMCA activity as the responsible mechanism, as postsynaptic $\mathrm{Ca}^{2+}$ influx may be as much as 100 -fold greater than that on the presynaptic side, depending on the permeability of glutamate receptors to $\mathrm{Ca}^{2+}$ relative to $\mathrm{Na}^{+}$(Lu et al., 2016).

In two previous studies, extracellular $\mathrm{pH}$-sensitive fluorescent dyes and proteins in brain slice preparations revealed transient acidification in response to presynaptic stimulation, before more substantial alkalinization (Krishtal et al., 1987; Du et al., 2014). In both studies, there is room to argue that the signal from the cleft of these conventional neuronal synapses was drowned out by the signal from the much larger extrasynaptic volume, but it would seem even less plausible that the acidic transient arose outside the cleft. Our experiments revealed no signs of acidification in response to a single AP at the Drosophila NMJ, or in response to bursts of APs at the Drosophila NMJ or the mouse calyx of Held synapse. Significantly, line scan data collected on a CLSM at rates up to 560 lines per second showed no sign of an initial acidic transient at the Drosophila NMJ. However, we should accept that there may be certain classes of conventional neuronal synapses with a high probability of release per AZ, and a low reliance on PMCA, where acidification develops into a measurable signal at the onset of activity.

On the face of it, the release of protons into the synaptic cleft should result in cleft acidification. However, we can expect acidification to be ameliorated by the rapid reactions within the phosphate and bicarbonate buffering systems, and the rapid diffusion and dilution of protons and their reaction products into the substantially larger volume of the synaptic cleft. A model based on a reaction-diffusion scheme is required to adequately accommodate this complexity, but even a rudimentary consideration suggests that the lifetime of any acid transient will be extremely short. The lateral extent of the synaptic cleft is substantially greater than the distance between presynaptic and postsynaptic membranes, and so diffusion within the cleft might be reduced to a $2 \mathrm{D}$ problem. Sites of release (AZs) can be placed at the nodes of an infinite square lattice, and given coordinates $\left(X_{i}=i * L, y_{j}=j * L\right)$, where $i$ and $j$ are integers and $\mathrm{L}(0.8 \mu \mathrm{m})$ is the distance between adjacent AZs. The bulk of the protons available to be released from an SV will not be free in the lumen, but rather, bound to glutamate (Cho and von Gersdorff, 2014), and possibly bound to ATP coloaded with glutamate. The time scale of the acid transient can be approximated by the time scale of $2 \mathrm{D}$ diffusion $(\tau)$, which can be calculated by $\tau=\frac{\left(\mathrm{L}_{R M S}\right)^{2}}{4 * D}$, where $\mathrm{L}_{R M S}$ is the root mean square distance between AZs and D is the effective diffusion coefficient, including all of the buffers (Berg, 1993). In the case when an AP triggers release at every $\mathrm{AZ}$, that is, where $\mathrm{L}_{\mathrm{RMS}}$ is 0.57 $\mu \mathrm{m}$, a range of diffusion coefficients on the order of $10^{-5} \frac{\mathrm{cm}^{2}}{\mathrm{~s}}$ $\leftarrow$

Cac-kisser-pHerry-TM examined at a live NMJ on a confocal microscope. $\boldsymbol{H}$, Examination of presynaptic Cac-kisser-pHerry-TM at a fixed NMJ. Antibodies were applied for the neuronal plasma membrane ( $\alpha$ HRP-Dylight 405), the AZ protein Brp (nc82-AF555), and SE-pHluorin ( $\alpha$ GFP-AF488). 
(Vanysek, 2002; Somersalo et al., 2012) will result in $\tau$ on the order of tens to hundreds of microseconds. The amplitude may be substantial, but it will largely dissipate before the arrival of the following AP in a burst, and therefore unlikely to influence STSP. In contrast, cleft alkalinization, as a consequence of $\mathrm{Ca}^{2+}$ clearance as described in this manuscript, will largely persist until the arrival of a following AP at any burst frequency greater than $\sim 5$ $\mathrm{Hz}$, providing the basis for the potentiation of release or reception of neurotransmitters.

\section{References}

Arosio D, Ricci F, Marchetti L, Gualdani R, Albertazzi L, Beltram F (2010) Simultaneous intracellular chloride and $\mathrm{pH}$ measurements using a GFPbased sensor. Nat Methods 7:516-518.

Barnes S, Bui Q (1991) Modulation of calcium-activated chloride current via $\mathrm{pH}$-induced changes of calcium channel properties in cone photoreceptors. J Neurosci 11:4015-4023.

Beckwith-Cohen B, Holzhausen LC, Wang TM, Rajappa R, Kramer RH (2019) Localizing proton-mediated inhibitory feedback at the retinal horizontal cell-cone synapse with genetically encoded $\mathrm{pH}$ probes. J Neurosci 39:651-662.

Benetti F, Micetic I, Carsughi F, Spinozzi F, Bubacco L, Beltramini M (2011) Insights into the oligomerization process of the $\mathrm{C}$-terminal domain of human plasma membrane $\mathrm{Ca}(2)^{+}$-ATPase. Arch Biochem Biophys 506: $194-200$.

Berg H (1993) Random walks in biology, revised ed. Princeton, NJ: Princeton UP.

Borst JG, Soria van Hoeve J (2012) The calyx of held synapse: from model synapse to auditory relay. Annu Rev Physiol 74:199-224.

Brand AH, Perrimon N (1993) Targeted gene expression as a means of altering cell fates and generating dominant phenotypes. Development 118: 401-415.

Brini M, Carafoli E, Calì T (2017) The plasma membrane calcium pumps: focus on the role in (neuro)pathology. Biochem Biophys Res Commun 483:1116-1124.

Chakrabarti R, Wichmann C (2019) Nanomachinery organizing release at neuronal and ribbon synapses. Int J Mol Sci 20:E2147.

Chen HY, Chesler M (2015) Autocrine boost of NMDAR current in hippocampal CA1 pyramidal neurons by a PMCA-dependent, perisynaptic, extracellular pH shift. J Neurosci 35:873-877.

Chen TW, Wardill TJ, Sun Y, Pulver SR, Renninger SL, Baohan A, Schreiter ER, Kerr RA, Orger MB, Jayaraman V, Looger LL, Svoboda K, Kim DS (2013) Ultrasensitive fluorescent proteins for imaging neuronal activity. Nature 499:295-300.

Chen ZL, Huang RQ (2014) Extracellular pH modulates GABAergic neurotransmission in rat hypothalamus. Neuroscience 271:64-76.

Chesler M (2003) Regulation and modulation of pH in the brain. Physiol Rev 83:1183-1221.

Cho S, von Gersdorff H (2014) Proton-mediated block of $\mathrm{Ca}^{2+}$ channels during multivesicular release regulates short-term plasticity at an auditory hair cell synapse. J Neurosci 34:15877-15887.

Chouhan AK, Zhang J, Zinsmaier KE, Macleod GT (2010) Presynaptic mitochondria in functionally different motor neurons exhibit similar affinities for $\mathrm{Ca}^{2+}$ but exert little influence as $\mathrm{Ca}^{2+}$ buffers at nerve firing rates in situ. J Neurosci 30:1869-1881.

Dana H, Mohar B, Sun Y, Narayan S, Gordus A, Hasseman JP, Tsegaye G, Holt GT, Hu A, Walpita D, Patel R, Macklin JJ, Bargmann CI, Ahrens MB, Schreiter ER, Jayaraman V, Looger LL, Svoboda K, Kim DS (2016) Sensitive red protein calcium indicators for imaging neural activity. Elife 5:e12727.

Davenport CM, Detwiler PB, Dacey DM (2008) Effects of pH buffering on horizontal and ganglion cell light responses in primate retina: evidence for the proton hypothesis of surround formation. J Neurosci 28:456-464.

Desai SA, Lnenicka GA (2011) Characterization of postsynaptic $\mathrm{Ca}^{2+}$ signals at the Drosophila larval NMJ. J Neurophysiol 106:710-721.

DeVries SH (2001) Exocytosed protons feedback to suppress the $\mathrm{Ca}^{2+}$ current in mammalian cone photoreceptors. Neuron 32:1107-1117.

Di Leva F, Domi T, Fedrizzi L, Lim D, Carafoli E (2008) The plasma membrane $\mathrm{Ca}^{2+}$ ATPase of animal cells: structure, function and regulation. Arch Biochem Biophys 476:65-74.

Du J, Reznikov LR, Price MP, Zha XM, Lu Y, Moninger TO, Wemmie JA,
Welsh MJ (2014) Protons are a neurotransmitter that regulates synaptic plasticity in the lateral amygdala. Proc Natl Acad Sci U S A 111:89618966.

Fahrenfort I, Steijaert M, Sjoerdsma T, Vickers E, Ripps H, van Asselt J, Endeman D, Klooster J, Numan R, ten Eikelder H, von Gersdorff $\mathrm{H}$, Kamermans M (2009) Hemichannel-mediated and pH-based feedback from horizontal cells to cones in the vertebrate retina. PLoS One 4:e6090.

Fedirko N, Svichar N, Chesler M (2006) Fabrication and use of high-speed, concentric $\mathrm{h}+-$ and $\mathrm{Ca}^{2+}$-selective microelectrodes suitable for in vitro extracellular recording. J Neurophysiol 96:919-924.

Fedirko N, Avshalumov M, Rice ME, Chesler M (2007) Regulation of postsynaptic $\mathrm{Ca}^{2+}$ influx in hippocampal CA1 pyramidal neurons via extracellular carbonic anhydrase. J Neurosci 27:1167-1175.

González-Inchauspe C, Urbano FJ, Di Guilmi MN, Uchitel OD (2017) Acid-sensing ion channels activated by evoked released protons modulate synaptic transmission at the mouse calyx of held synapse. J Neurosci 37:2589-2599.

Gottfried JA, Chesler M (1996) Temporal resolution of activity-dependent pH shifts in rat hippocampal slices. J Neurophysiol 76:2804-2807.

Gratz SJ, Goel P, Bruckner JJ, Hernandez RX, Khateeb K, Macleod GT, Dickman D, O'Connor-Giles KM (2019) Endogenous tagging reveals differential regulation of $\mathrm{Ca}(2+)$ channels at single active zones during presynaptic homeostatic potentiation and depression. J Neurosci 39: 2416-2429.

Groh A, de Kock CP, Wimmer VC, Sakmann B, Kuner T (2008) Driver or coincidence detector: modal switch of a corticothalamic giant synapse controlled by spontaneous activity and short-term depression. J Neurosci 28:9652-9663.

Guerrero G, Reiff DF, Agarwal G, Ball RW, Borst A, Goodman CS, Isacoff EY (2005) Heterogeneity in synaptic transmission along a Drosophila larval motor axon. Nat Neurosci 8:1188-1196.

Heckmann M, Dudel J (1998) Evoked quantal currents at neuromuscular junctions of wild type Drosophila larvae. Neurosci Lett 256:77-80.

Highstein SM, Holstein GR, Mann MA, Rabbitt RD (2014) Evidence that protons act as neurotransmitters at vestibular hair cell-calyx afferent synapses. Proc Natl Acad Sci U S A 111:5421-5426.

Hirasawa H, Yamada M, Kaneko A (2012) Acidification of the synaptic cleft of cone photoreceptor terminal controls the amount of transmitter release, thereby forming the receptive field surround in the vertebrate retina. J Physiol Sci 62:359-375.

Johansen J, Halpern ME, Johansen KM, Keshishian H (1989) Stereotypic morphology of glutamatergic synapses on identified muscle cells of Drosophila larvae. J Neurosci 9:710-725.

Kim MH, Korogod N, Schneggenburger R, Ho WK, Lee SH (2005) Interplay between $\mathrm{Na}^{+} / \mathrm{Ca}_{2}{ }^{+}$exchangers and mitochondria in $\mathrm{Ca}^{2+}$ clearance at the calyx of held. J Neurosci 25:6057-6065.

Klose MK, Chu D, Xiao C, Seroude L, Robertson RM (2005) Heat shockmediated thermoprotection of larval locomotion compromised by ubiquitous overexpression of Hsp70 in Drosophila melanogaster. J Neurophysiol 94:3563-3572.

Kraig RP, Ferreira-Filho CR, Nicholson C (1983) Alkaline and acid transients in cerebellar microenvironment. J Neurophysiol 49:831-850.

Krishtal OA, Osipchuk YV, Shelest TN, Smirnoff SV (1987) Rapid extracellular $\mathrm{pH}$ transients related to synaptic transmission in rat hippocampal slices. Brain Res 436:352-356.

Lnenicka GA, Grizzaffi J, Lee B, Rumpal N (2006) $\mathrm{Ca}^{2+}$ dynamics along identified synaptic terminals in Drosophila larvae. J Neurosci 26:1228312293.

Lu Z, Chouhan AK, Borycz JA, Lu Z, Rossano AJ, Brain KL, Zhou Y, Meinertzhagen IA, Macleod GT (2016) High-probability neurotransmitter release sites represent an energy-efficient design. Curr Biol 26:2562-2571.

Lujan B, Kushmerick C, Banerjee TD, Dagda RK, Renden R (2016) Glycolysis selectively shapes the presynaptic action potential waveform. J Neurophysiol 116:2523-2540.

Lujan B, Dagostin A, von Gersdorff H (2019) Presynaptic diversity revealed by $\mathrm{Ca}(2+)$-permeable AMPA receptors at the calyx of held synapse. J Neurosci 39:2981-2994.

Macleod GT, Hegström-Wojtowicz M, Charlton MP, Atwood HL (2002) Fast calcium signals in Drosophila motor neuron terminals. J Neurophysiol 88:2659-2663.

Macleod GT, Marin L, Charlton MP, Atwood HL (2004) Synaptic vesicles: test for a role in presynaptic calcium regulation. J Neurosci 24:2496-2505. 
Makani S, Chesler M (2007) Endogenous alkaline transients boost postsynaptic NMDA receptor responses in hippocampal CA1 pyramidal neurons. J Neurosci 27:7438-7446.

Makani S, Chesler M (2010a) Rapid rise of extracellular pH evoked by neural activity is generated by the plasma membrane calcium ATPase. J Neurophysiol 103:667-676.

Makani S, Chesler M (2010b) Barium plateau potentials of CA1 pyramidal neurons elicit all-or-none extracellular alkaline shifts via the plasma membrane calcium ATPase. J Neurophysiol 104:1438-1444.

Makani S, Chen HY, Esquenazi S, Shah GN, Waheed A, Sly WS, Chesler M (2012) NMDA receptor-dependent afterdepolarizations are curtailed by carbonic anhydrase 14: regulation of a short-term postsynaptic potentiation. J Neurosci 32:16754-16762.

Melom JE, Akbergenova Y, Gavornik JP, Littleton JT (2013) Spontaneous and evoked release are independently regulated at individual active zones. J Neurosci 33:17253-17263.

Meyer AC, Neher E, Schneggenburger R (2001) Estimation of quantal size and number of functional active zones at the calyx of held synapse by nonstationary EPSC variance analysis. J Neurosci 21:7889-7900.

Miesenböck G, De Angelis DA, Rothman JE (1998) Visualizing secretion and synaptic transmission with $\mathrm{pH}$-sensitive green fluorescent proteins. Nature 394:192-195.

National Research Council (2011) Guide for the care and use of laboratory animals: Eighth edition. Washington, DC: The National Academies Press.

Newman ZL, Hoagland A, Aghi K, Worden K, Levy SL, Son JH, Lee LP, Isacoff EY (2017) Input-specific plasticity and homeostasis at the Drosophila larval neuromuscular junction. Neuron 93:1388-1404.e10.

Obara M, Szeliga M, Albrecht J (2008) Regulation of $\mathrm{pH}$ in the mammalian central nervous system under normal and pathological conditions: facts and hypotheses. Neurochem Int 52:905-919.

Paalasmaa P, Taira T, Voipio J, Kaila K (1994) Extracellular alkaline transients mediated by glutamate receptors in the rat hippocampal slice are not due to a proton conductance. J Neurophysiol 72:2031-2033.

Palmer MJ, Hull C, Vigh J, von Gersdorff H (2003) Synaptic cleft acidification and modulation of short-term depression by exocytosed protons in retinal bipolar cells. J Neurosci 23:11332-11341.

Pawlu C, DiAntonio A, Heckmann M (2004) Postfusional control of quantal current shape. Neuron 42:607-618.

Pérez Koldenkova VP, Nagai T (2013) Genetically encoded Ca(2+) indicators: properties and evaluation. Biochim Biophys Acta 1833:1787-1797.

Peters A, Palay SL, Webster HD (1991) The fine structure of the nervous system. New York: Harper and Row.

Rossano AJ, Chouhan AK, Macleod GT (2013) Genetically encoded pHindicators reveal activity-dependent cytosolic acidification of Drosophila motor nerve termini in vivo. J Physiol 591:1691-1706.

Rossano AJ, Kato A, Minard KI, Romero MF, Macleod GT (2017) Na(+) $/ \mathrm{H}(+)$ exchange via the Drosophila vesicular glutamate transporter mediates activity-induced acid efflux from presynaptic terminals. J Physiol 595:805-824.

Rowland KC, Irby NK, Spirou GA (2000) Specialized synapse-associated structures within the calyx of held. J Neurosci 20:9135-9144.

Sandstrom DJ (2011) Extracellular protons reduce quantal content and prolong synaptic currents at the Drosophila larval neuromuscular junction. J Neurogenet 25:104-114.

Sankaranarayanan S, De Angelis D, Rothman JE, Ryan TA (2000) The use of pHluorins for optical measurements of presynaptic activity. Biophys J 79:2199-2208.

Shaner NC, Campbell RE, Steinbach PA, Giepmans BN, Palmer AE, Tsien RY (2004) Improved monomeric red, orange and yellow fluorescent proteins derived from discosoma sp. red fluorescent protein. Nat Biotechnol 22:1567-1572.
Shcherbakova DM, Verkhusha VV (2013) Near-infrared fluorescent proteins for multicolor in vivo imaging. Nat Methods 10:751-754.

Shemiakina II, Ermakova GV, Cranfill PJ, Baird MA, Evans RA, Souslova EA, Staroverov DB, Gorokhovatsky AY, Putintseva EV, Gorodnicheva TV, Chepurnykh TV, Strukova L, Lukyanov S, Zaraisky AG, Davidson MW, Chudakov DM, Shcherbo D (2012) A monomeric red fluorescent protein with low cytotoxicity. Nat Commun 3:1204.

Singh M, Denny H, Smith C, Granados J, Renden R (2018) Presynaptic loss of dynamin-related protein 1 impairs synaptic vesicle release and recycling at the mouse calyx of held. J Physiol 596:6263-6287.

Sinning A, Hübner CA (2013) Minireview: pH and synaptic transmission. FEBS Lett 587:1923-1928.

Somersalo E, Occhipinti R, Boron WF, Calvetti D (2012) A reactiondiffusion model of $\mathrm{CO}_{2}$ influx into an oocyte. J Theor Biol 309:185-203.

Stawarski M, Justs KA, Hernandez RX, Macleod GT (2018) The application of 'kisser' probes for resolving the distribution and microenvironment of membrane proteins in situ. J Neurogenet 32:236-245.

Stevens CF (2003) Neurotransmitter release at central synapses. Neuron 40:381-388.

Stewart BA, Atwood HL, Renger JJ, Wang J, Wu CF (1994) Improved stability of Drosophila larval neuromuscular preparations in haemolymphlike physiological solutions. J Comp Physiol A 175:179-191.

Sullivan KM, Scott K, Zuker CS, Rubin GM (2000) The ryanodine receptor is essential for larval development in Drosophila melanogaster. Proc Natl Acad Sci U S A 97:5942-5947.

Tang CM, Dichter M, Morad M (1990) Modulation of the N-methyl-Daspartate channel by extracellular $\mathrm{H}^{+}$. Proc Natl Acad Sci U S A 87: $6445-6449$.

Thomas RC (2009) The plasma membrane calcium ATPase (PMCA) of neurones is electroneutral and exchanges $2 \mathrm{H}^{+}$for each $\mathrm{Ca}^{2+}$ or $\mathrm{Ba}^{2+}$ ion extruded. J Physiol 587:315-327.

Tong CK, Chen K, Chesler M (2006) Kinetics of activity-evoked pH transients and extracellular $\mathrm{pH}$ buffering in rat hippocampal slices. J Neurophysiol 95:3686-3697.

Traynelis SF, Cull-Candy SG (1991) Pharmacological properties and $\mathrm{H}^{+}$ sensitivity of excitatory amino acid receptor channels in rat cerebellar granule neurones. J Physiol 433:727-763.

Vanysek P (2002) Ionic conductivity and diffusion at infinite dilution. In: CRC handbook of chemistry and physics (Lide DR, ed), pp 76-78. Boca Raton, FL: CRC.

Vincent PF, Cho S, Tertrais M, Bouleau Y, von Gersdorff H, Dulon D (2018) Clustered $\mathrm{Ca}(2+)$ channels are blocked by synaptic vesicle proton release at mammalian auditory ribbon synapses. Cell Rep 25:3451-3464.e3.

von Gersdorff H, Vardi E, Matthews G, Sterling P (1996) Evidence that vesicles on the synaptic ribbon of retinal bipolar neurons can be rapidly released. Neuron 16:1221-1227.

Vroman R, Klaassen LJ, Howlett MH, Cenedese V, Klooster J, Sjoerdsma T, Kamermans M (2014) Extracellular ATP hydrolysis inhibits synaptic transmission by increasing $\mathrm{pH}$ buffering in the synaptic cleft. PLoS Biol 12:e1001864.

Wang TM, Holzhausen LC, Kramer RH (2014) Imaging an optogenetic pH sensor reveals that protons mediate lateral inhibition in the retina. Nat Neurosci 17:262-268.

Wemmie JA, Taugher RJ, Kreple CJ (2013) Acid-sensing ion channels in pain and disease. Nat Rev Neurosci 14:461-471.

Zhao H, Carney KE, Falgoust L, Pan JW, Sun D, Zhang Z (2016) Emerging roles of $\mathrm{Na}(+) / \mathrm{H}(+)$ exchangers in epilepsy and developmental brain disorders. Prog Neurobiol 138:19-35.

Zhou C, Xiao C, Deng C, Hong Ye J (2007) Extracellular proton modulates GABAergic synaptic transmission in rat hippocampal CA3 neurons. Brain Res 1145:213-220. 